\title{
Aspirin-Mediated Attenuation of Intervertebral Disc Degeneration by Ameliorating Reactive Oxygen Species In Vivo and In Vitro
}

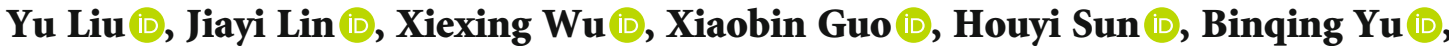 \\ Jining Shen $\mathbb{D}$, Jiaxiang Bai $\mathbb{D}$, Zhanghuan Chen $\mathbb{D}$, Huilin Yang, Dechun Geng $\mathbb{D}$, \\ and Haiqing Mao
}

Department of Orthopaedics, The First Affiliated Hospital of Soochow University, Suzhou 215006, China

Correspondence should be addressed to Dechun Geng; szgengdc@163.com and Haiqing Mao; maohq@suda.edu.cn

Received 9 June 2019; Revised 4 September 2019; Accepted 14 September 2019; Published 6 November 2019

Academic Editor: Fabiana Morroni

Copyright (C) 2019 Yu Liu et al. This is an open access article distributed under the Creative Commons Attribution License, which permits unrestricted use, distribution, and reproduction in any medium, provided the original work is properly cited.

Intervertebral disc (IVD) degeneration (IDD) is a major cause of low back pain. The pathogenesis of IDD is associated with the disturbance of reactive oxygen species (ROS) equilibrium, inflammation, and matrix loss. Aspirin is a nonsteroidal antiinflammatory drug that effectively inhibits inflammation and oxidative stress and has been widely used for the treatment of back pain. Therefore, we hypothesize that aspirin reverses the IDD process via antioxidative and anti-inflammatory effects on the AMPK signaling pathway. In vitro, aspirin diminished cellular oxygen free radicals (ROS, nitric oxide (NO)) and inflammatory cytokines (interleukin- (IL-) $1 \beta$ and IL-6 and tumor necrosis factor alpha (TNF- $\alpha$ )) induced by lipopolysaccharides (LPS) in nucleus pulposus cells (NPCs). We found that aspirin preserved the extracellular matrix (ECM) content of collagen type II (COL2) and aggrecan while inhibiting the expression of matrix-degenerating enzymes, including matrix metalloproteinase 3 and 13 (MMP-3 and MMP-13) and A disintegrin and metalloproteinase with thrombospondin motifs 4 and 5 (ADAMTS-4, ADAMTS-5). Aspirin significantly promoted the ratios of p-AMPK to AMPK and p-ACC to ACC expression in NPCs. Furthermore, pretreatment with the AMPK inhibitor compound $\mathrm{C}$ abrogated the antioxidant effects of aspirin. In vivo, an IDD model was established in Sprague-Dawley rats via percutaneous disc puncture with the 20-gauge needle on levels 8-9 and 9-10 of the coccygeal vertebrae. Imaging assessment showed that after aspirin treatment, improvements in disc height index (DHI) ranged from 1.22-fold to 1.54-fold and nucleus pulposus signal strength improved from 1.26-fold to 1.33-fold. Histological analysis showed that aspirin treatment prevented the loss of COL2 and decreased MMP-3 and MMP-13, inducible NO synthase (iNOS), cyclooxygenase-2 (COX-2), IL-1 $\beta$, and TNF- $\alpha$ expression in the IVD tissues. These results suggest that treatment with aspirin could reverse the IDD process via the AMPK signaling pathway, which provides new insights into the potential clinical applications of aspirin, particularly for IDD treatment.

\section{Introduction}

In the past few decades, the prevalence of degenerative disc diseases has increased, and low back pain is now a major health problem $[1,2]$. Researchers have suggested that more than half of musculoskeletal disability cases are caused by degenerative disc diseases. The United States spends more than $\$ 100$ billion every year for the treatment of degenerative disc diseases [3]. Currently, relevant clinical therapy for degenerative disc diseases mainly includes surgery and conservative treatment, which are aimed at achieving symptomatic relief rather than preventing intervertebral disc (IVD) degeneration (IDD) [4].

The pathogenesis of IDD remains unclear, but it is typically characterized by matrix dehydration in the nucleus pulposus, loss of disc height, and mechanical dysfunction [5-7], thereby resulting in traumatic injury and pain. Some investigators have found that multiple pathological changes can 
enhance the levels of inflammatory cytokines, such as interleukin-1 $\beta$ (IL-1 $\beta$ ) and tumor necrosis factor alpha (TNF$\alpha$ ). These cytokines can increase the expression of A disintegrin and metalloproteinase with thrombospondin motifs 4 and 5 (ADAMTS-4, ADAMTS-5) and matrix metalloproteinase 3 and 13 (MMP-3, MMP-13) and promote aggrecan degradation [8, 9]. Associations among herniation, IDD, and the inflammatory response have been well established [9]. The IDD tissue contains many inflammatory cells which secrete TNF- $\alpha$, IL- $1 \beta$, and other cytokines found in the tissue at the injury site [10]. Furthermore, these inflammatory cytokines are upregulated with age and IDD progression and are positively correlated with the degree of pain [11]. Importantly, the expression of inflammatory cytokines is not only associated with degeneration but is also considered as its cause.

Recent research has suggested that oxidative stress plays an important role in the development of degeneration [12-14]. During oxidative stress, excessive amounts of reactive oxygen species (ROS), such as superoxide anions, hydroxyl radicals, and hydrogen peroxide, are generated by the mitochondria [15]. Excessive ROS levels can directly impair nucleus pulposus cells (NPCs), interfere with the homeostasis of the IVD matrix, reduce the synthesis of proteoglycans, and increase the expression of MMPs $[16,17]$. ROS and chronic inflammation are interdependent processes that have been correlated with degeneration [18]. Research indicates that ROS can promote the expression of inflammatory cytokines in the degenerated tissue [19]. Therefore, decreasing the expression of ROS could alleviate IDD.

Aspirin is a salicylate that was introduced in the late 1890 s and has been used for the treatment of a variety of inflammatory diseases [20]. Traditionally, aspirin has been utilized to reduce the risk of cardiovascular disease and in the treatment of rheumatism or arthritis [21]. It has been recently reported in several studies that a lowdose aspirin administration can prevent preeclampsia [22], inhibit platelets to increase $\mathrm{T}$ cell activity [23], and attenuate ROS expression in microglia $[24,25]$. It has also been suggested that aspirin could reduce oxidative stress and inflammation, which in turn may reverse the IDD process.

The present study induced oxidative stress and inflammation in NPCs using lipopolysaccharides (LPS) to assess the capability of aspirin to improve IDD based on its antioxidant activity and possible relevant signaling pathways. Our results clearly show that aspirin could significantly improve IDD via its antioxidative and anti-inflammatory effects in vivo and in vitro. Importantly, our data indicated that aspirin exerts its protective effects through the $5^{\prime}$ adenosine monophosphate-activated protein kinase (AMPK) signaling pathway. These results improve our understanding of the underlying mechanism by which aspirin inhibits IDD.

\section{Materials and Methods}

2.1. Ethics Statement. All of the experiments performed in this study were approved by the Ethics Committee of the
First Affiliated Hospital of Soochow University (Suzhou, China) (No. 201801A005). All of the animal surgical interventions and treatments and animal care were conducted in strict accordance with the directives of the Laboratory Animal Center of Soochow University, Suzhou, China.

2.2. Experimental Animals. We purchased Sprague-Dawley (SD) rats at osseous maturity (male, age: 3 months, weight: $450 \mathrm{~g} \pm 50 \mathrm{~g}$ ) from the Laboratory Animal Center of Soochow University. The animals were kept in a ventilated environment with a $12 \mathrm{~h} / 12 \mathrm{~h}$ light-dark cycle at a constant temperature of $21^{\circ} \mathrm{C}$.

2.3. Reagents and Antibodies. We purchased aspirin (V900185), LPS (Escherichia coli 0111:B4), and phosphatebuffered saline (PBS) from Sigma-Aldrich (St. Louis, Missouri, USA). A $2^{\prime}, 7^{\prime}$-dichlorodihydrofluorescein diacetate (DCFH-DA) fluorescent probe (Beyotime Institute of Biotechnology, Shanghai, China) was used to assess intracellular ROS production, and 5-aminoimidazole-4-carboxamide ribonucleotide (AICAR) and compound $\mathrm{C}$ were purchased from Selleck Chemicals (Shanghai, China). Additionally, we purchased rabbit anti-rat collagen type II (COL2; ab34712), MMP-3 (ab13533), MMP-13 (ab39012), IL-1 $\beta$ (ab9722), TNF- $\alpha$ (ab6671), AMPK (ab32047), phospho-AMPK (pAMPK; ab133448), acetyl-CoA carboxylase (ACC; ab45174), phospho-ACC (p-ACC, ab68191), nitric oxide (NO) synthase (iNOS; ab15323), cyclooxygenase-2 (COX-2; ab15191), and nuclear factor (erythroid-derived 2)-like 2 (Nrf-2; ab137550) antibodies, as well as goat anti-rabbit immunoglobulin G (IgG) heavy+light chain (H\&L) Alexa Fluor 488 (ab150077) and Alexa Fluor 647 (ab150079) from Abcam (Cambridge, UK). Anti- $\beta$-actin and $4^{\prime}, 6$-diamidino2-phenylindole (DAPI) were purchased from Beyotime. Aggrecan (bs-1223R), ADAMTS-4 (bs-4191R), and ADAMTS-5 (bs-3573R) were purchased from Bioss (Beijing, China).

2.4. NPC Culture. NPCs derived from normal human individuals are difficult to obtain and are usually obtained from teenagers who developed vertebral body fracture bursts. Different types and degrees of IDD largely influence the traits of the obtained NPCs. For these reasons, in this study, we used Sprague-Dawley (SD) rat NPCs. SD rats were given an overdose of sodium pentobarbital $(100 \mathrm{mg} / \mathrm{kg})$ and then sacrificed. The nucleus pulposus was carefully separated from the annular fibrous tissues and collected from the caudal discs (Co1-Co5) under aseptic conditions and a Stemi 305 dissecting microscope (Zeiss, Oberkochen, Germany). The tissues were dissociated using $0.5 \%(w / v)$ collagenase type II for $2 \mathrm{~h}$ at $37^{\circ} \mathrm{C}$. Next, the digested tissues were cultured in Dulbecco's modified Eagle's medium/Nutrient Mixture F12 (DMEM/F-12; Invitrogen, Waltham, MA, USA) supplemented with $15 \%$ fetal bovine serum (FBS; Invitrogen) and antibiotics (1\% penicillin and streptomycin) in an incubator maintained at $5 \%$ carbon dioxide and at $37^{\circ} \mathrm{C}$. Upon reaching confluency, the cells were harvested using $0.25 \%$ trypsinethylenediaminetetraacetic acid (EDTA; Invitrogen). The medium was changed every $3 \mathrm{~d}$. The NPCs were subsequently 
divided into five experimental groups. (1) Control group: the NPCs were treated with DMEM/F-12 for $24 \mathrm{~h}$. (2) LPS group: the NPCs were cultured in DMEM/F-12 with LPS $(1 \mu \mathrm{g} / \mathrm{mL})$ for $24 \mathrm{~h}$. (3) Aspirin+LPS group: the NPCs were pretreated with DMEM/F-12 supplemented with aspirin (5 or 25 $\mu \mathrm{g} / \mathrm{mL}$ ) for $3 \mathrm{~h}$ and then cultured in DMEM/F-12 with LPS $(1 \mu \mathrm{g} / \mathrm{mL})$ and aspirin $(5$ or $25 \mu \mathrm{g} / \mathrm{mL})$ for $24 \mathrm{~h}$. (4) Aspirin +LPS+compound C (AMPK inhibitor) group: the NPCs were pretreated with compound $\mathrm{C}(100 \mu \mathrm{M})$ for $24 \mathrm{~h}$, then with DMEM/F-12 supplemented with aspirin $(25 \mu \mathrm{g} / \mathrm{mL})$ for $3 \mathrm{~h}$, and finally with DMEM/F-12 with LPS $(1 \mu \mathrm{g} / \mathrm{mL})$ and aspirin $(25 \mu \mathrm{g} / \mathrm{mL})$ for $24 \mathrm{~h}$. (5) LPS+AICAR (AMPK agonist) group: the NPCs were pretreated with AICAR (500 $\mu \mathrm{M})$ for $24 \mathrm{~h}$, then cultured with DMEM/F-12 supplemented with LPS $(1 \mu \mathrm{g} / \mathrm{mL})$ for $24 \mathrm{~h}$.

2.5. Cell Viability Assay. To assess the effects of different reagents, we seeded NPCs (density: $3 \times 10^{3}$ cells per well) in a 96-well plate and viability was examined using a Cell Counting Kit-8 (CCK-8; Dojindo Co., Kumamoto, Japan) following the manufacturer's protocols. The NPCs were treated with different concentrations of aspirin and LPS for different time periods. Optical density (OD) was measured at a wavelength of $450 \mathrm{~nm}$ using a Gen5 microplate reader (BioTek Instruments, Inc., Winooski, VT, USA).

2.6. Measurement of ROS Production. NPCs (density: $1 \times 10^{5}$ cells per well) in a 24-well plate were pretreated with different concentrations of aspirin and incubated with $1 \mu \mathrm{g} / \mathrm{mL}$ LPS. Subsequently, these were incubated with $10 \mu \mathrm{M}$ DCFH-DA for $30 \mathrm{~min}$ in the dark. The cells were then assessed in terms of ROS-mediated fluorescence under an inverted fluorescence microscope (IFM) and by flow cytometry (FCM) using fluorescein isothiocyanate (FITC) [26]. Under the FITC fluorescence channel in IFM, typical positive cells were labeled as bright green. The average fluorescence intensity can be calculated by counting by FCM in the FITC channel and for comparison.

2.7. Assessment of NO Production. We measured NO using a nitrite detection kit (Beyotime Institute of Biotechnology, Jiangsu, China). Briefly, we collected a $50 \mu \mathrm{L}$ sample of the cell culture medium and mixed this with $50 \mu \mathrm{L}$ each of Griess Reagents I and II from the kit. We then measured the absorbance of the mixture at a wavelength of $540 \mathrm{~nm}$ and calculated nitrite concentration using a standard curve of sodium nitrite [27].

2.8. Enzyme-Linked Immunosorbent Assay (ELISA). We determined IL- $1 \beta$, IL- 6 , and TNF- $\alpha$ levels in the cell culture supernatants using an ELISA kit (Hangzhou MultiSciences Biotech Co. Ltd., Hangzhou, China). Briefly, the NPCs were cultured to confluency in 24-well plates (density: $5 \times 10^{4}$ cells per well) and pretreated with or without aspirin, followed by incubation with LPS $(1 \mu \mathrm{g} / \mathrm{mL})$. At the end of the treatment period, we transferred $100 \mu \mathrm{L}$ aliquots of cell culture media to wells coated with anti-IL-1, anti-IL-6, or anti-TNF- $\alpha$ antibody for $2 \mathrm{~h}$. Then, the wells were thoroughly washed, followed by the addition of $50 \mu \mathrm{L}$ of the E-selectin antibody to each well and incubation for $45 \mathrm{~min}$ to allow coating of the well. After incubation, the wells were immediately washed, and a peroxidase-conjugated secondary polyclonal antibody solution was added to each well and incubated for $30 \mathrm{~min}$. After washing to remove any unbound antibodies, we added a substrate solution to each well and allowed the reaction to develop for $20 \mathrm{~min}$. Color development was then stopped, and absorbance was measured at a wavelength of $450 \mathrm{~nm}$ using a microplate reader.

2.9. Reverse Transcription Quantitative Polymerase Chain Reaction (RT-qPCR). We extracted total ribonucleic acid (RNA) using TRIzol reagent (Invitrogen), and then total RNA concentrations were determined using a NanoDrop 2000 (Thermo Fisher Scientific, Waltham, MA, USA). For reverse transcription, the PrimeScript RT Master Mix kit (Takara, Kusatsu, Japan) was used, and $1 \mu \mathrm{g}$ of reverse transcription product (cDNA) was used for PCR amplification. PCR amplification required $20 \mu \mathrm{L}$ of the reverse transcription product in each reaction, plus $10 \mu \mathrm{L}$ Forget-Me-Not qPCR Master Mix (Biotium, Inc., Hayward, CA, USA), $0.5 \mu \mathrm{L}$ of each primer, $1 \mu \mathrm{L}$ of $\mathrm{cDNA}$, and $8 \mu \mathrm{L}$ RNase-free distilled water $\left(\mathrm{dH}_{2} \mathrm{O}\right.$; Invitrogen). The reactions were performed using the CFX96 Touch Real-Time PCR Detection System (Bio-Rad Laboratories, Hercules, CA, USA). Cycle threshold values were normalized to the level of glyceraldehyde 3phosphate dehydrogenase (GAPDH). To calculate messenger RNA levels, we used the $2^{-\Delta \Delta C T}$ method [28]. The primers sequences are presented in Supplemental Table 1.

2.10. Western Blot Assay. Total protein extraction was performed in a radioimmunoprecipitation assay (RIPA) supplemented with phenylmethanesulfonyl fluoride (PMSF), and protein concentration was measured with a bicinchoninic acid (BCA) kit (Beyotime, Shanghai). Approximately $30 \mu \mathrm{g}$ of protein was separated via sodium dodecyl sulfate polyacrylamide gel electrophoresis (SDS-PAGE) and transferred onto polyvinylidene difluoride (PVDF) membranes (BioRad). After blocking with 5\% nonfat milk, the membranes were incubated with primary antibodies against $\beta$-actin $(1: 5,000)$, AMPK $(1: 500)$, p-AMPK $(1: 500)$, ACC $(1: 1,000), \quad$-ACC $(1: 1,000)$, iNOS $(1: 250)$, COX-2 $(1: 1,000)$, and $\mathrm{Nrf}-2(1: 1,000)$ overnight at $4^{\circ} \mathrm{C}$, followed by the respective secondary antibodies. Finally, we detected bands using the Electrochemiluminescence Plus Reagent (Thermo Fisher) and quantified their intensity with Image Lab software version 3.0 (Bio-Rad).

2.11. Immunocytochemical (ICC) Staining. Cells were cultured on 24-well plates. After washing with PBS, the cells were fixed with formaldehyde for $30 \mathrm{~min}$ and incubated in $2 \%$ fetal calf serum for $2 \mathrm{~h}$. Then, the cells were incubated overnight with COL2 $(1: 200)$, aggrecan $(1: 200)$, MMP-3 (1:200), MMP-13 (1:200), ADAMTS-4 (1:150), and ADAMTS-5 $(1: 150)$ rabbit anti-rat antibodies and then with goat anti-rabbit IgG H\&L Alexa Fluor 488 or $647(1: 1,000)$ for $2 \mathrm{~h}$, followed by counterstaining with DAPI for $30 \mathrm{~min}$.

2.12. Surgical Procedures. After $12 \mathrm{~h}$ of fasting and $4 \mathrm{~h}$ of water deprivation, we anesthetized the animals via intraperitoneal injection of $0.3 \%$ pentobarbital. An IDD model was 
established as described by Han et al.'s [29] method and by adopting a standard surgical procedure. The areas between the eighth and ninth coccygeal vertebrae (Co8-Co9) and between the ninth and tenth vertebrae (Co9-Co10) were punctured using a 20-gauge needle. To ensure degeneration, we punctured the annulus fibrosus and then rotated the needle for $5 \mathrm{~s}$ and held it steady for $30 \mathrm{~s}$. To avoid possible influences of individual differences, the following groups were established: (1) control group: nonpunctured Co7-Co8 discs; (2) aspirin group: punctured $\mathrm{Co} 8-\mathrm{Co} 9$ discs and injected aspirin; and (3) IDD group: punctured Co9-Co10 discs and injected PBS. Additionally, we randomly divided the aspirin-injected rats into subgroups that received low $(10 \mu \mathrm{g} / \mathrm{mL} ; n=20)$ and high $(100 \mu \mathrm{g} / \mathrm{mL} ; n=20)$ concentrations of aspirin. Similar to our previous research, we eliminated the influences of injected volume by injecting only $2 \mu \mathrm{L}$ of PBS or high- or low-concentration aspirin into the center space of the nucleus pulposus at a depth of $6 \mathrm{~mm}$ $[30,31]$. To avoid secondary damage caused by puncture, all of the injections were performed with a 33-gauge Hamilton syringe (Hamilton Co., Reno, NV, USA) 3 days after the day of puncture.

2.13. Histological Analysis. We fixed the rat caudal discs with $4 \%$ paraformaldehyde for $48 \mathrm{~h}$ and decalcified these in $10 \%$ EDTA for 45 days. The fixed Co7-Co10 samples then underwent paraffin embedding. Sections ( $5 \mu \mathrm{m}$ in thickness) were cut and then stained with either hematoxylin and eosin (H\&E) or Safranin O Fast Green. We determined the histological grade according to Masuda [32].

2.14. Immunohistochemistry (IHC) Staining. After dewaxing, gradient dehydration, and antigen retrieval, we selected representative indicators of inflammation (IL-1 $\beta$ (1:100), TNF- $\alpha(1: 150))$, oxidative stress (iNOS $(1: 100)$, COX-2 $(1: 100))$, and degeneration (COL2 $(1: 200)$ and MMP-3 $(1: 150))$, added their respective primary antibodies to the sections, and incubated these in the dark at $4^{\circ} \mathrm{C}$ for $12 \mathrm{~h}$. Then, the sections were incubated at room temperature in a buffer with secondary antibodies for $35 \mathrm{~min}$. After incubation, the sections were rinsed and then counterstained with hematoxylin. We quantified each group using Image ProPlus image analysis software version 6.0 (Media Cybernetics, Rockville, MD, USA).

2.15. X-Ray and Magnetic Resonance Imaging (MRI) Analysis. Seven days after the initial annulus puncture, each group of rats $(n=20)$ was randomly selected to undergo Xray $(n=10)$ and MRI $(n=10)$ scans before these were sacrificed. The rats were kept in a supine position with their tails straight for placement on a molybdenum target radiographic-image unit (General Electric, Boston, MA, USA). Radiographs were taken at a collimator-to-film distance of $66 \mathrm{~cm}$, an exposure of $63 \mathrm{mAs}$, and a penetration power of $35 \mathrm{kV}$. We performed MRI using a $1.5 \mathrm{~T}$ system (GE) to obtain T2-weighted images (repletion time: 3,000 ms; echo time: $80 \mathrm{~ms}$; field of view: $200 \mathrm{~mm}^{2}$; slice thickness: $1.4 \mathrm{~mm}$ ) on the coronal plane. All of the radiographic images were saved in a Neusoft PACS/RIS DICOM
3.0 medical imaging system (Neusoft, Liaoning, China). IVD height and the adjacent upper and lower vertebralbody heights were measured using Neusoft PACS/RIS measuring tools; from these values, the disc height index (DHI) was calculated.

2.16. Statistical Analysis. We used SPSS software version 25 (SSPS, Inc., Chicago, IL, USA) for statistical analysis. Data were expressed in terms of fold change, and experiments were conducted independently at least thrice. Statistical significance was determined by using one-way ANOVA and Tukey's honest significant-difference (HSD) tests. Differences in data were further analyzed between each group; mean differences were expressed with $95 \%$ confidence intervals (CIs). Differences were considered statistically significant at $P<0.05$.

\section{Results}

3.1. Aspirin Attenuates Oxidative Stress and Expression of Proinflammatory Cytokines in NPCs. LPS stimulated ROS expression in a dose- and time-dependent manner without toxic effects (Supp. Figs. S1A-D), significantly upregulated MMP-3, and inhibited COL2 in NPCs (Supp. Fig. S1E). LPS also upregulated iNOS and COX-2 and downregulated Nrf-2 (Supp. Figs. S1F-I). These results were concordant to the findings of previous studies $[33,34]$.

Figure 1 (a) shows that aspirin $(<100 \mu \mathrm{g} / \mathrm{mL})$ is not toxic to NPCs. Both aspirin dosages significantly mitigated ROS expression within the range of $32.9 \%(5 \mu \mathrm{g} / \mathrm{mL})$ to $56.4 \%$ $(25 \mu \mathrm{g} / \mathrm{mL})$ compared to the LPS group $(P<0.05$; Figures 1(b) and 1(c)). Western blotting showed that aspirin significantly decreased protein expression of iNOS $(P<0.05)$ and COX-2 $(P<0.05)$. In addition, aspirin treatment increased the expression of Nrf-2 $(P<0.05)$, which is a key protein involved in antioxidant responses (Figures $1(\mathrm{~d})-$ $1(\mathrm{~g}))$.

In response to oxidative stress, NPCs secrete a series of inflammatory cytokines. Our results indicated that IL- $1 \beta$, IL-6, and TNF- $\alpha$ expression markedly increased following LPS induction. Specifically, IL-1 $\beta$ expression increased by $\sim 2.3$-fold (Figure 1(h)), IL-6 increased by $\sim 3.9$-fold (Figure 1(i)), and TNF- $\alpha$ increased by $\sim 2.8$-fold $(P<0.05)$ (Figure $1(\mathrm{j})$ ). Treatment with aspirin reversed the elevated expression of these inflammatory cytokines (Figures 1(h)$1(j))$. We also evaluated the extracellular concentration of NO, which increased $\sim 12.2$-fold $(P<0.05)$ after LPS intervention. Aspirin reversed this decrease (Figure $1(\mathrm{k}))$.

3.2. Aspirin Alleviates LPS-Induced NPC Dysfunction. RTPCR analysis indicated that LPS reduced the expression of COL2 and aggrecan $(P<0.05$; Figures $2(\mathrm{a})$ and $2(\mathrm{~b}))$, whereas it increased that of MMP-3 and MMP-13 $(P<0.05$ ; Figures 2(c) and 2(d)) and the upstream regulatory factors ADAMTS-4 and ADAMTS-5 of MMPs $(P<0.05$; Figures 2(e) and 2(f)). Aspirin reversed these changes (Figures 2(a)-2(f)). ICC staining showed that COL2 had a greater distribution around the nucleus in NPCs than in the LPS group and is full of aggrecan. In addition, the expression 


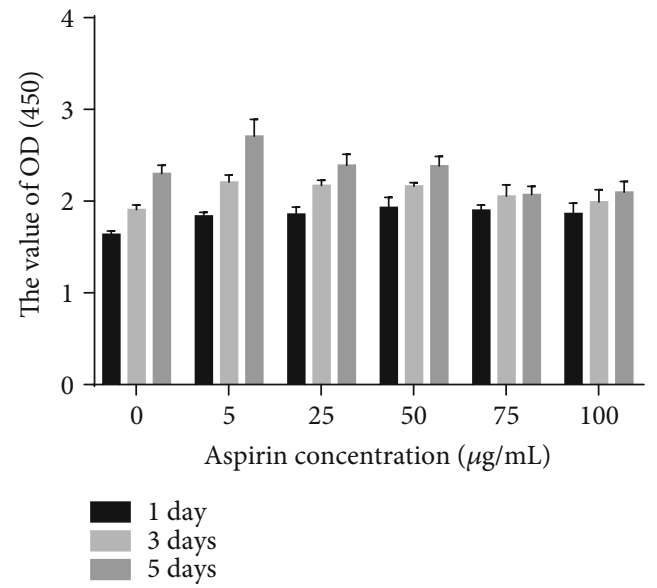

(a)
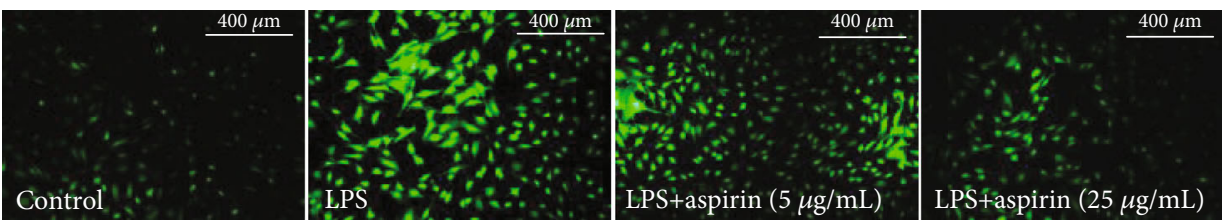

(b)

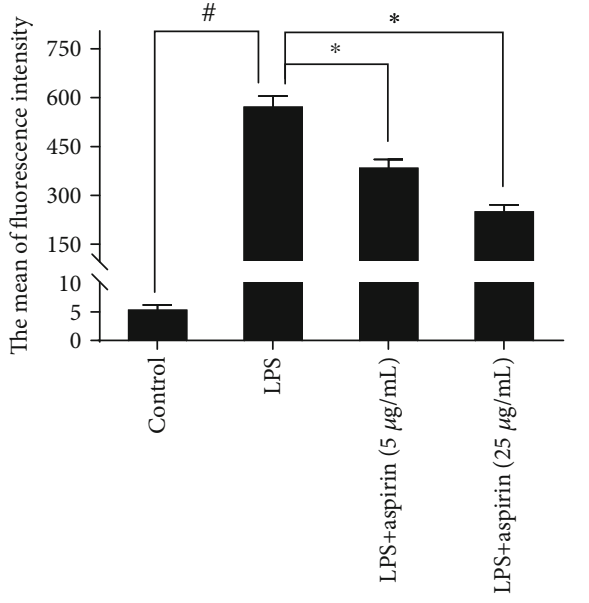

(c)

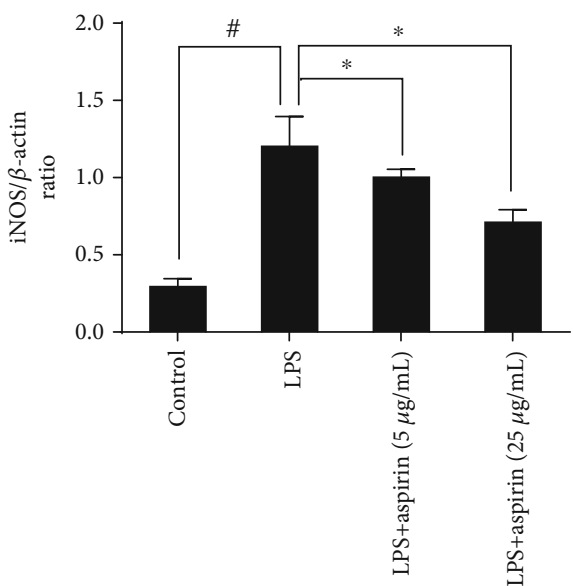

(e)

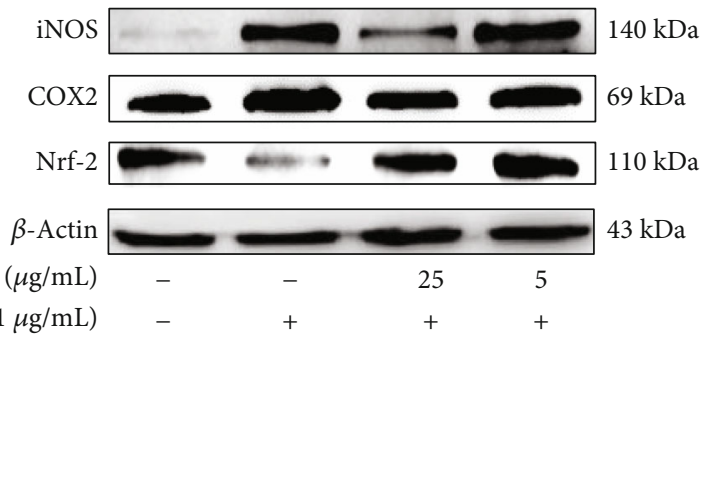

(d)

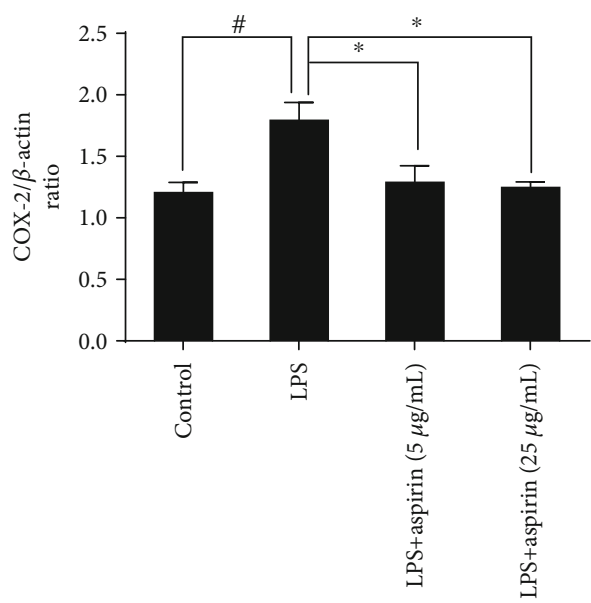

(f)

FIgURE 1: Continued. 


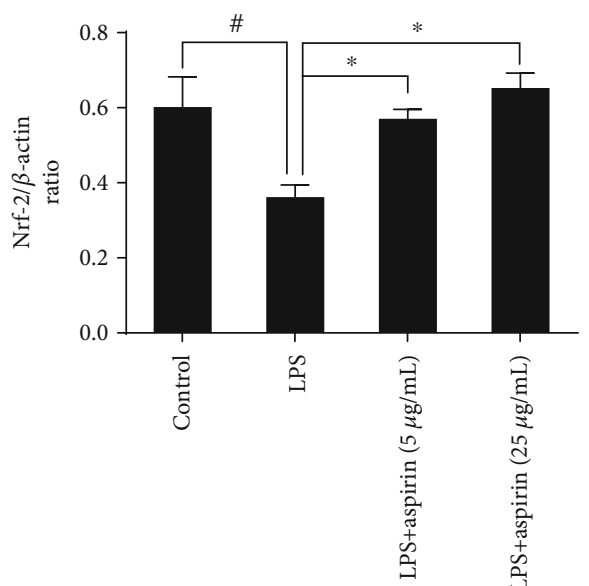

$(\mathrm{g})$

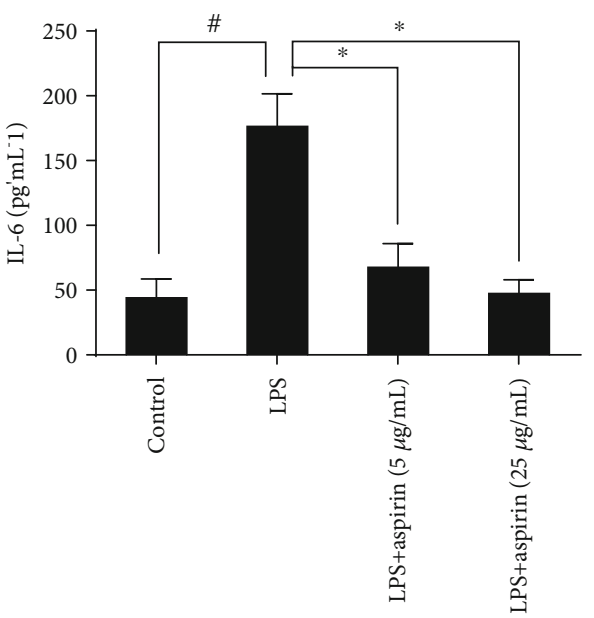

(i)

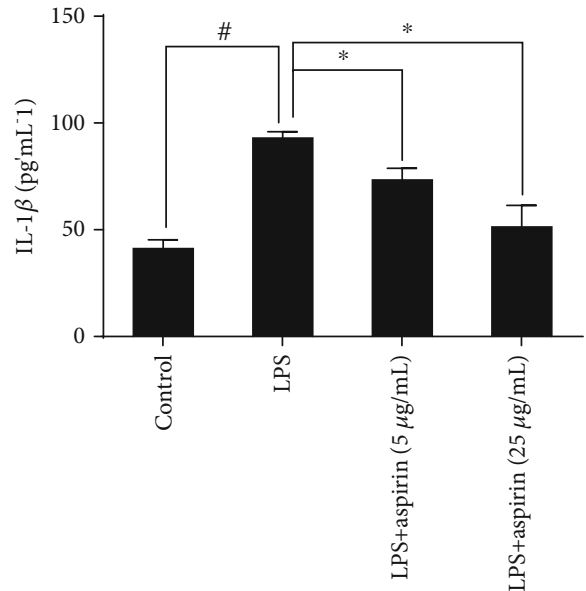

(h)

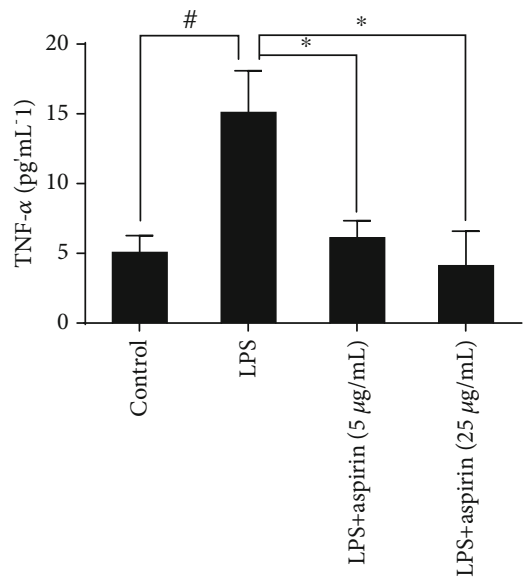

(j)

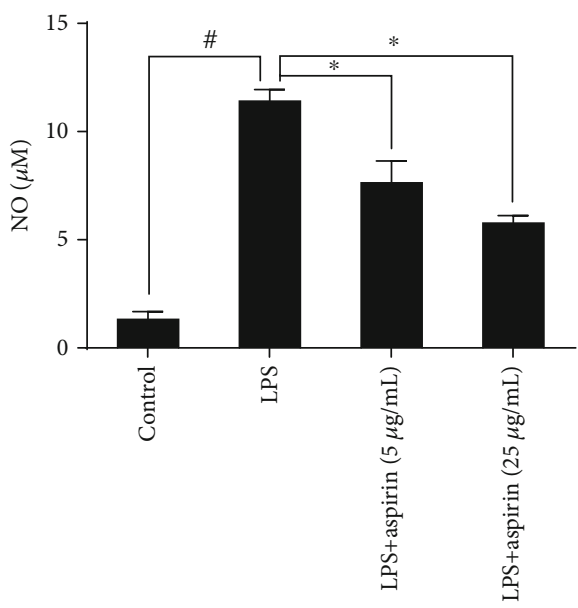

(k)

FIGURE 1: Aspirin attenuates oxidative stress and inflammation in NPCs. (a) NPCs were cultured with different concentrations of aspirin (0, 5 , $25,50,75$, and $100 \mu \mathrm{g} / \mathrm{mL})$ for 1,3 , and $5 \mathrm{~d}$; cell viability was detected by CCK- $8\left({ }^{*} P<0.05\right.$ vs. control group). NPCs were pretreated with aspirin $(5$ or $25 \mu \mathrm{g} / \mathrm{mL}$ ) for $3 \mathrm{~h}$ and then stimulated by $1 \mu \mathrm{g} / \mathrm{mL}$ LPS for $24 \mathrm{~h}$. (b) Fluorescence images show ROS levels in different groups. Scale bar, $400 \mu \mathrm{m}$. (c) Average fluorescence intensity of ROS detected by FCM $\left({ }^{\#} P<0.05\right.$ vs. control group; ${ }^{*} P<0.05$ vs. LPS group). (d-g) Western blotting results of the iNOS, COX-2, and Nrf-2 ( $P<0.05$ vs. control group; ${ }^{*} P<0.05$ vs. LPS group). (h-j) ELISA-detected inflammatory cytokines IL- $\beta$, IL-6, and TNF- $\alpha$. (k) NO levels in different groups ( $P<0.05 v s$. control group; ${ }^{*} P<0.05$ vs. LPS group). ${ }^{\#} P$ and ${ }^{*} P<0.05$ by one-way ANOVA and Tukey's HSD test were further analyzed between LPS group vs. control group, aspirin $(5 \mu \mathrm{g} / \mathrm{mL})$ group $v s$. LPS group, and aspirin $(25 \mu \mathrm{g} / \mathrm{mL})$ group $v s$. LPS group $(n=3$ independent experiments). 


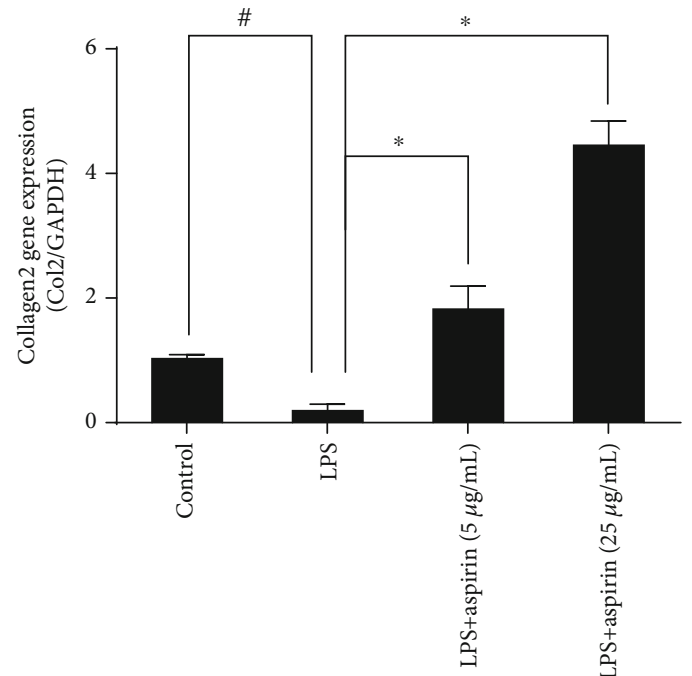

(a)

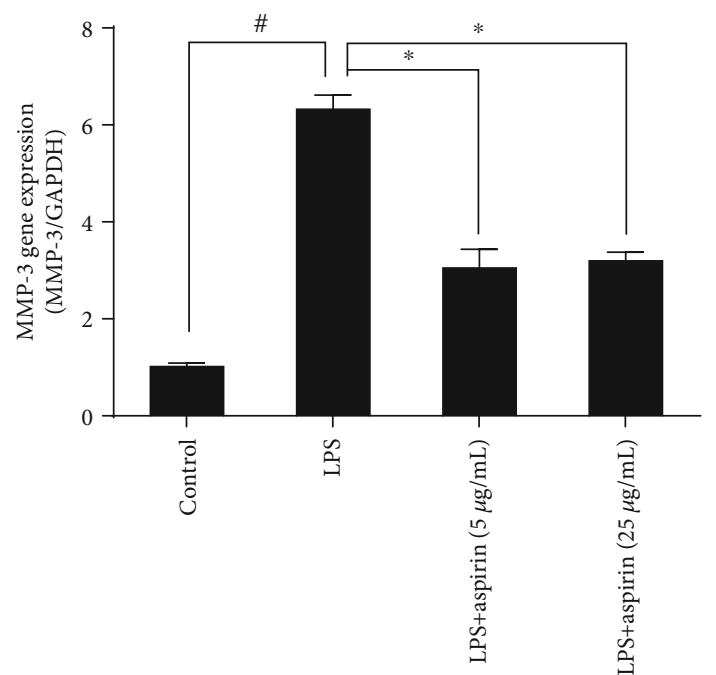

(c)

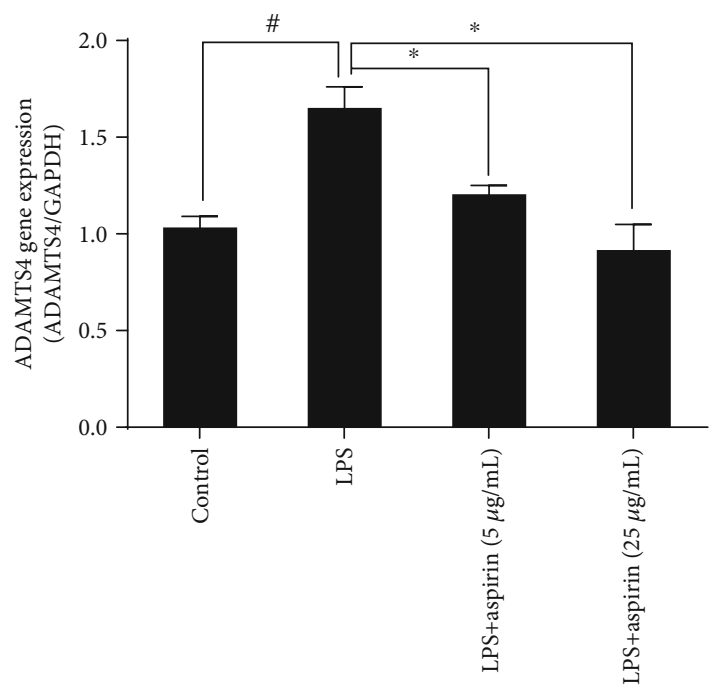

(e)

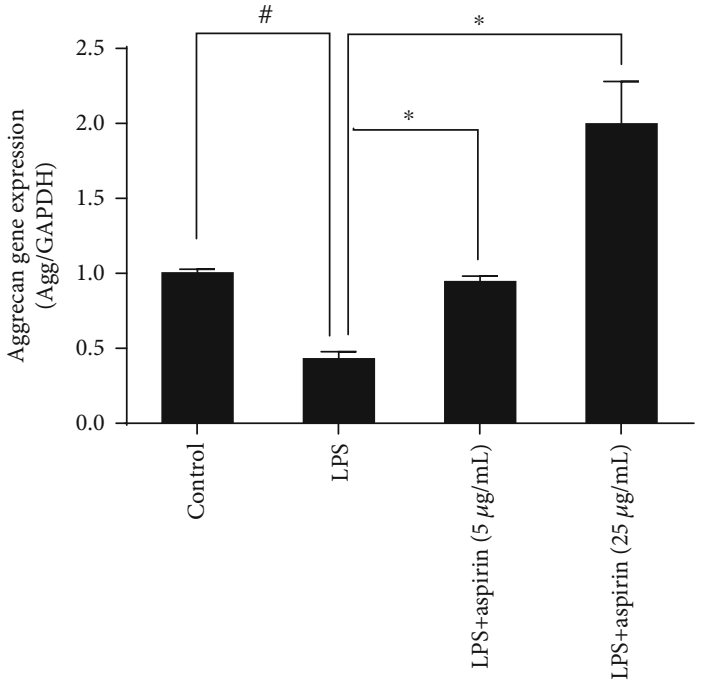

(b)

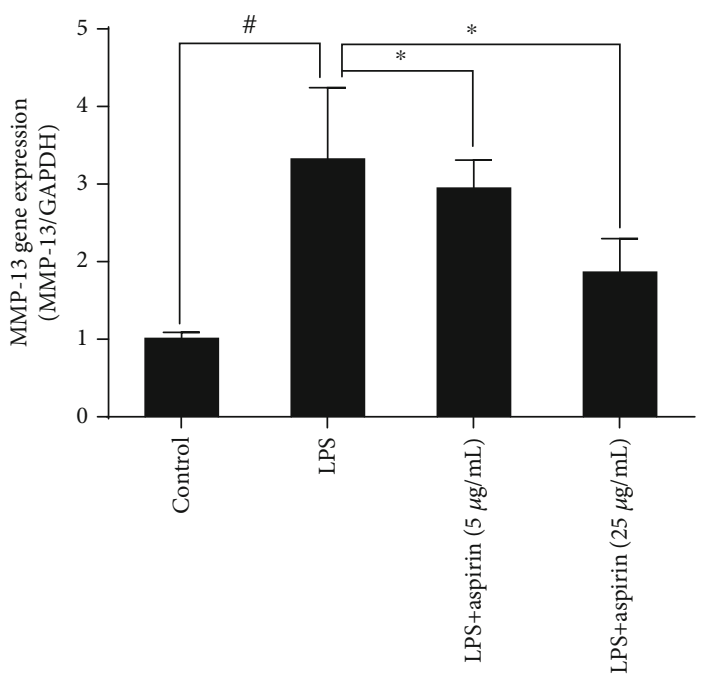

(d)

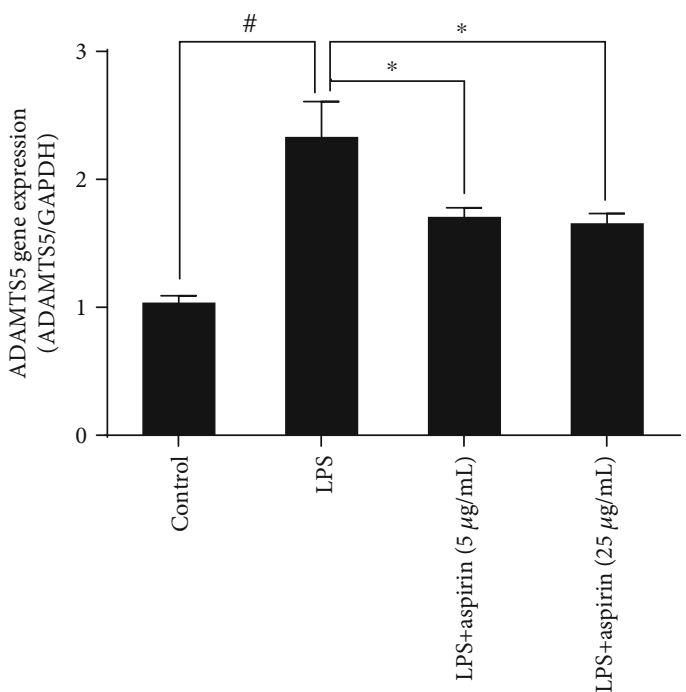

(f)

Figure 2: Continued 
Control
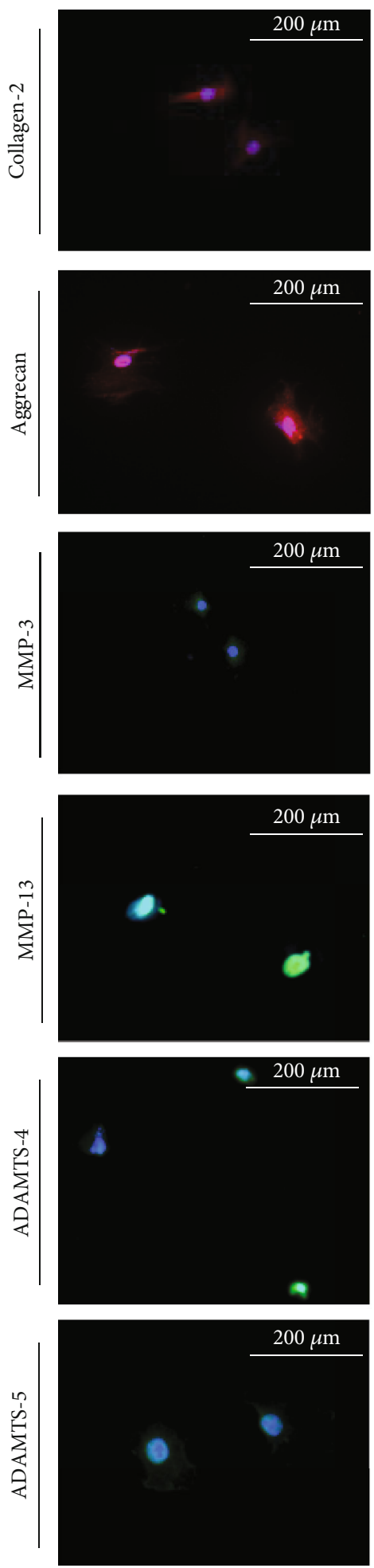

LPS
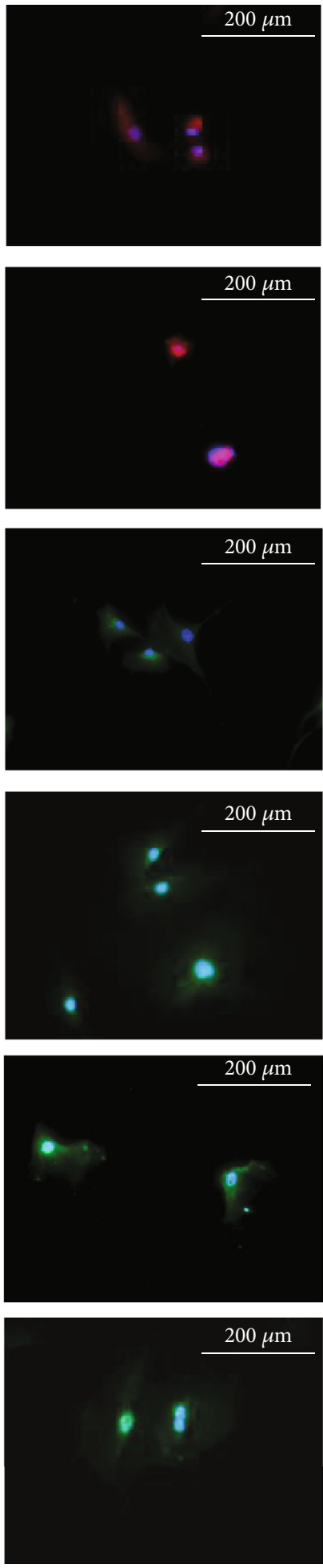

LPS+aspirin $(5 \mu \mathrm{g} / \mathrm{mL})$
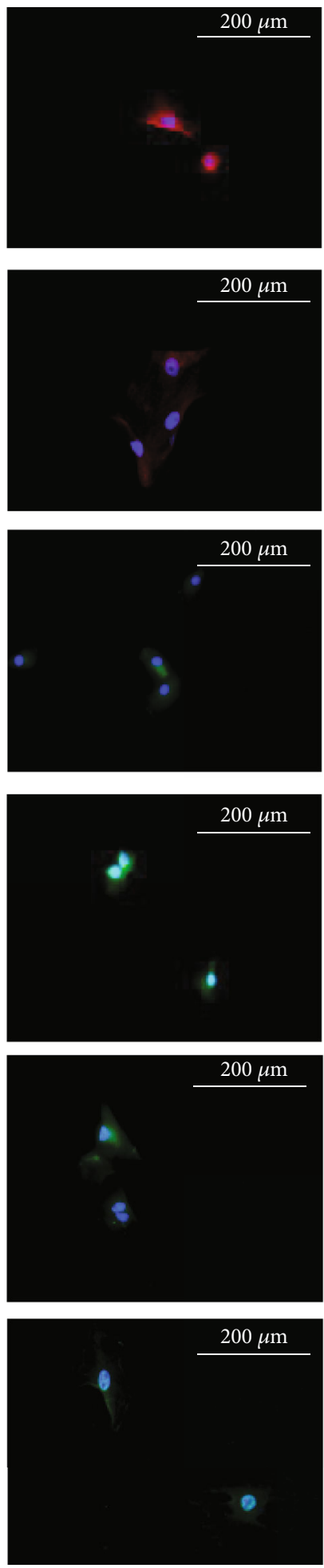

LPS+aspirin $(25 \mu \mathrm{g} / \mathrm{mL})$
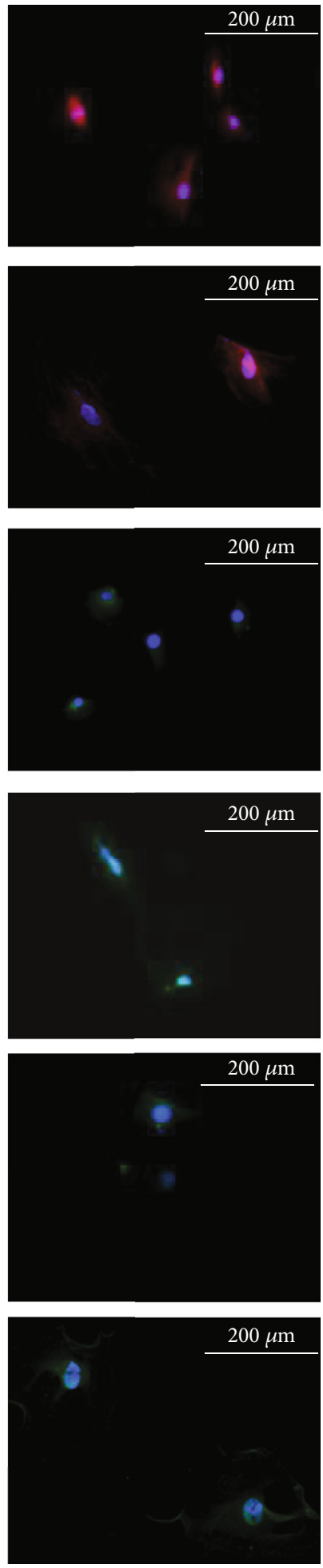

(g)

Figure 2: Aspirin alleviates LPS-induced degeneration of NPCs. NPCs were pretreated with aspirin ( 5 or $25 \mu \mathrm{g} / \mathrm{mL})$ for $3 \mathrm{~h}$, then stimulated by $1 \mu \mathrm{g} / \mathrm{mL}$ LPS for $24 \mathrm{~h}$. (a-f) RT-qPCR results for COL2, aggrecan, MMP-3, MMP-13, ADAMTS-4, and ADAMTS-5 (" $P<0.05$ vs. control group; ${ }^{*} P<0.05$ vs. LPS group). (g) Representative ICC image with COL2, aggrecan, MMP-3, MMP-13, ADAMTS-4, and ADAMTS-5 staining in NPCs. Scale bar, $200 \mu \mathrm{m} .{ }^{\#} P$ and ${ }^{*} P<0.05$ by one-way ANOVA and Tukey's HSD test were further analyzed between LPS group $v s$. control group, aspirin $(5 \mu \mathrm{g} / \mathrm{mL})$ group $v s$. LPS group, and aspirin $(25 \mu \mathrm{g} / \mathrm{mL})$ group $v s$. LPS group ( $n=3$ independent experiments). 


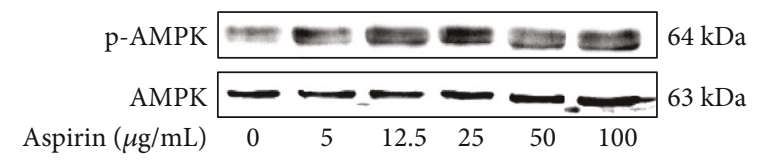

(a)

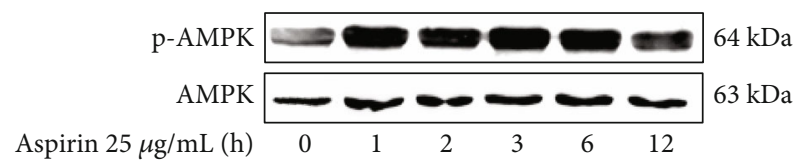

(c)

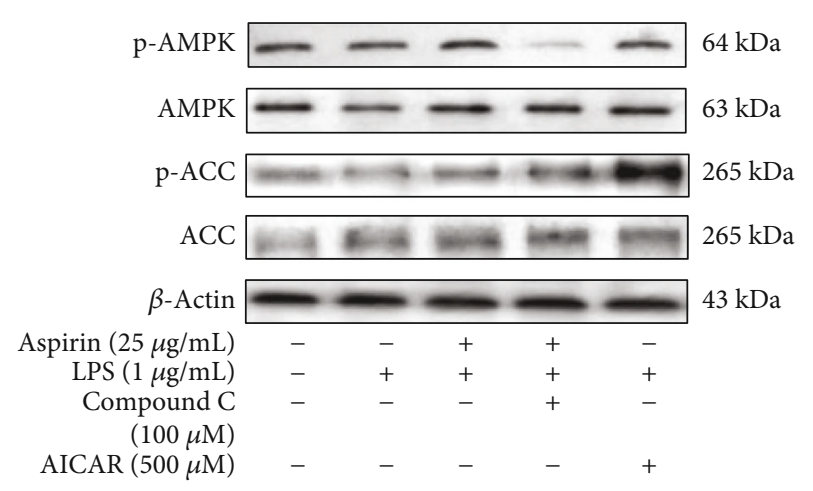

(e)

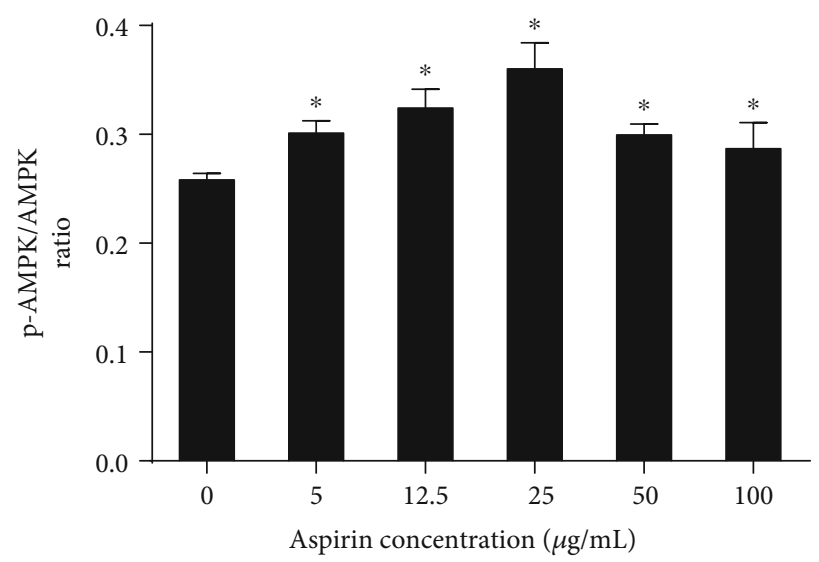

(b)

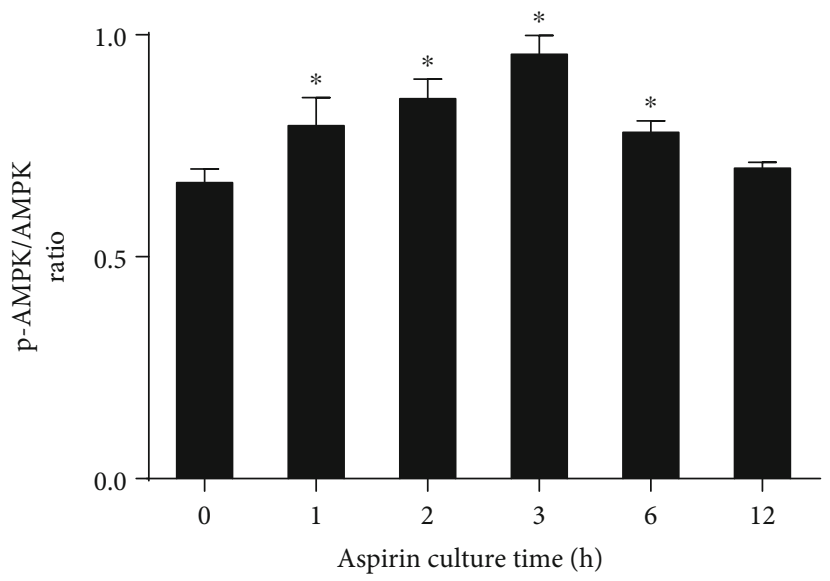

(d)

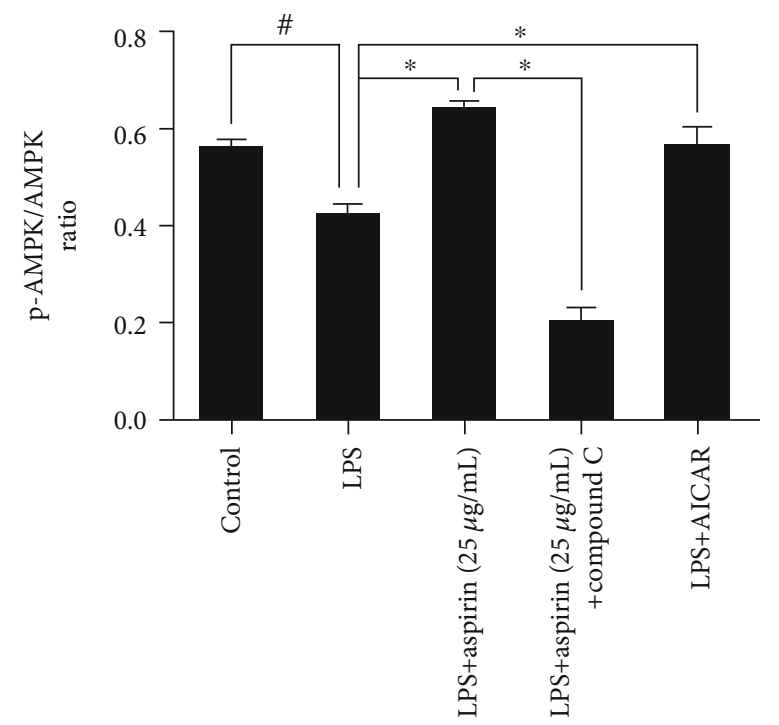

(f)

Figure 3: Continued. 


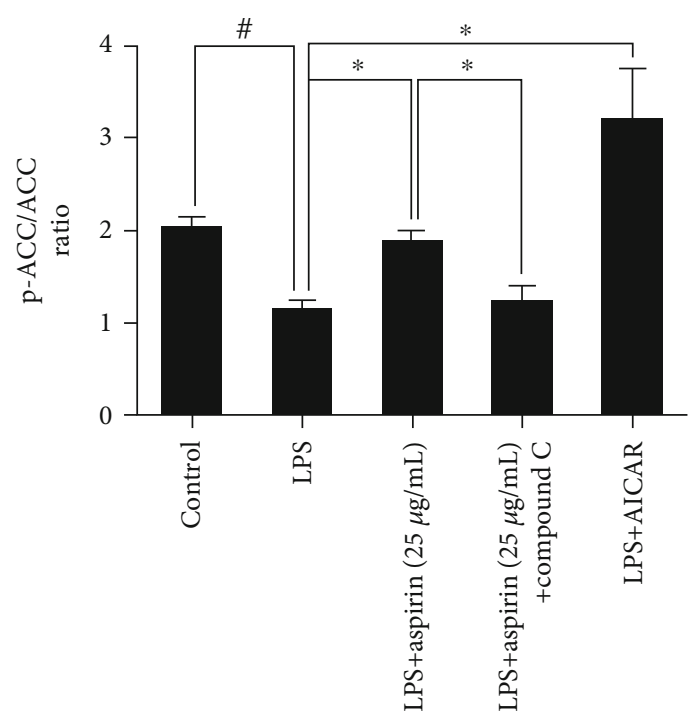

(g)

FIGURE 3: Aspirin activates the AMPK signaling pathway. NPCs were treated with different concentrations of aspirin $(0,5,12.5,25,50$, and $100 \mu \mathrm{g} / \mathrm{mL})$ for $3 \mathrm{~h}$. (a, b) Western blotting results for the p-AMPK/AMPK ratio $\left({ }^{*} P<0.05 v s\right.$. control group). NPCs were treated with aspirin $(25 \mu \mathrm{g} / \mathrm{mL})$ for different time periods $(0,1,2,3,6,12$, and $24 \mathrm{~h})$. (c, d) Western blotting results for the p-AMPK/AMPK ratio $\left({ }^{*} P<0.05 v s\right.$. control group). NPCs were pretreated with AMPK inhibitors (compound C, $100 \mu \mathrm{M}$ ) or AMPK agonists (AICAR, $500 \mu \mathrm{M}$ ) for $24 \mathrm{~h}$; aspirin $(25 \mu \mathrm{g} / \mathrm{mL})$ or LPS $(1 \mu \mathrm{g} / \mathrm{mL})$ was then added for $3 \mathrm{~h}$. $(\mathrm{e}-\mathrm{g})$ Western blot results for the $\mathrm{p}$-AMPK/AMPK and p-ACC/ACC ratios. ${ }^{\#} P$ and ${ }^{*} P<0.05$ by one-way ANOVA and Tukey's HSD test were further analyzed between LPS group vs. control group, aspirin $(25 \mu \mathrm{g} / \mathrm{mL})$ group $v s$. LPS group, aspirin $(25 \mu \mathrm{g} / \mathrm{mL})+\mathrm{LPS}+$ compound C group $v s$. aspirin $(25 \mu \mathrm{g} / \mathrm{mL})$ group, and LPS+AICAR group $v s$. LPS group ( $n=3$ independent experiments).

and distribution of MMP-3, MMP-13, ADAMTS-4, and ADAMTS-5 decreased after treatment with aspirin compared to the LPS group (Figure $2(\mathrm{~g})$ ).

3.3. Aspirin Plays a Protective Role via the AMPK Signaling Pathway. The AMPK signaling pathway is closely related to oxidative stress. We hypothesized that aspirin achieves its antioxidative stress effect by activating this pathway. Our results indicated that aspirin-induced AMPK phosphorylation is dose- (Figures 3(a) and 3(b)) and time-dependent (Figures 3(c) and 3(d)). In addition, when the aspirin dosage was $25 \mu \mathrm{g} / \mathrm{mL}$, the ratio of $\mathrm{p}$-AMPK to AMPK sharply increased, peaking at $3 \mathrm{~h}(P<0.05$; Figures $3(\mathrm{c})$ and $3(\mathrm{~d}))$. To further explore whether aspirin could activate AMPK, we detected the protein ACC, which is downstream of AMPK. Our data showed that treatment with aspirin markedly increased ACC phosphorylation compared to the LPS group $(P<0.05$; Figures 3(e)-3(g)).

After treatment with LPS, the $\mathrm{p}$-AMPK/APMK ratio was totally reversed $(P<0.05)$. Compound $\mathrm{C}$, which is a specific inhibitor of AMPK, significantly decreased $\mathrm{p}-\mathrm{AMPK}$ and $\mathrm{p}$ ACC (Supp. Figs. S2A-D). The effect of aspirin-activated AMPK signaling pathway was blocked after pretreatment with compound $\mathrm{C}(P<0.05$; Figures $3(\mathrm{e})-3(\mathrm{~g}))$. When we pretreated NPCs with compound $\mathrm{C}$ and then added aspirin to each group, the inhibition of ROS production was reversed $(P<0.05$; Figures $4(\mathrm{a})$ and 4(b)). Western blotting showed that compound $\mathrm{C}$ increased the protein expression levels of iNOS and COX-2 but inhibited the expression of Nrf-2 $(P<0.05$; Figures $4(\mathrm{c})-4(\mathrm{f}))$. Treatment with compound $\mathrm{C}$ and aspirin did not inhibit inflammatory cytokine expression
(Figures 4(g)-4(j)). RT-PCR analysis indicated that compound $\mathrm{C}$ increased the expression of MMP-3, MMP-13, ADAMTS-4, and ADAMTS-5 ( $P<0.05$; Figures 5(c)-5(f)), whereas it decreased the expression of COL2 and aggrecan $(P<0.05$; Figures 5(a) and 5(b)). ICC staining showed that COL2 and aggrecan expression decreased in NPCs, whereas that of MMP-3, MMP-13, ADAMTS-4, and ADAMTS-5 increased (Figure 6(a)). AICAR, which activates AMPK, reversed the ratio of $\mathrm{p}-\mathrm{AMPK}$ to $\mathrm{AMPK}$ after treatment with LPS and without aspirin $(P<0.05$; Figures $3(\mathrm{e})-3(\mathrm{~g}))$. AICAR plays a protective role against antioxidative stress by activating the AMPK signaling pathway. Taken together, these findings indicate that aspirin attenuates LPS-induced oxidative stress and ameliorates NPC dysfunction by activating the AMPK pathway.

3.4. Aspirin Ameliorates IDD in the In Vivo Rat Model. We successfully established an in vivo IDD model using SD rats $(n=20)$, then measured their degree of IDD by MRI $(n=10)$ and X-ray $(n=10)$. The MRI images obtained at the second week after puncture in the aspirin group had stronger T2-weighted signal intensities than the IDD group and ranged from $\sim 1.26$-fold to $\sim 1.33$-fold $(P<0.05$ ; Figures $7(\mathrm{a})$ and $7(\mathrm{~b})$ ). The change in IVD height was expressed by the DHI. We observed a significant improvement in the aspirin group compared to the IDD group, which ranged from $\sim 1.22$-fold to $\sim 1.54$-fold $(P<0.05$; Figures $7(\mathrm{c})$ and $7(\mathrm{~d}))$. During the experiment, the animals remained in good condition and did not die.

After H\&E (Figure 7(e)) and Safranin O Fast Green (Figure $7(\mathrm{f}))$ staining, the nucleus pulposus of the control 

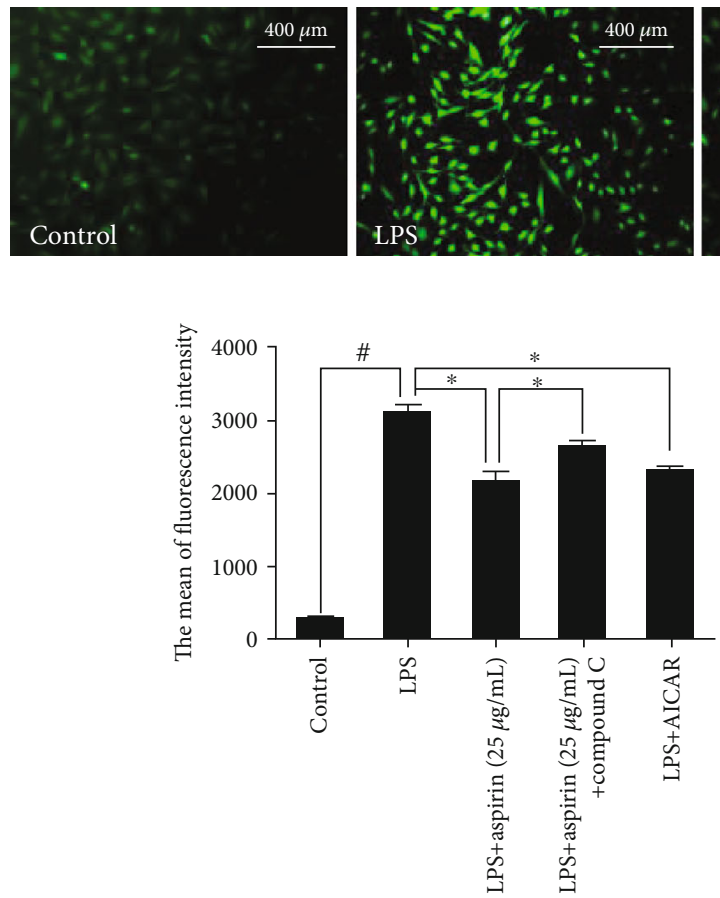

(b)

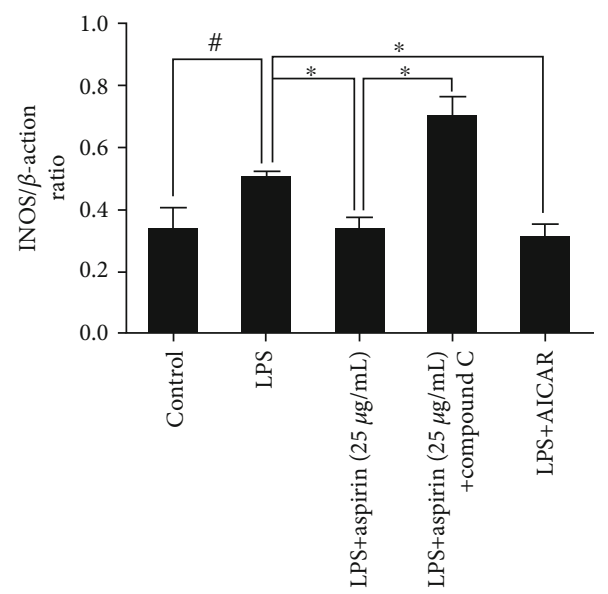

(d)
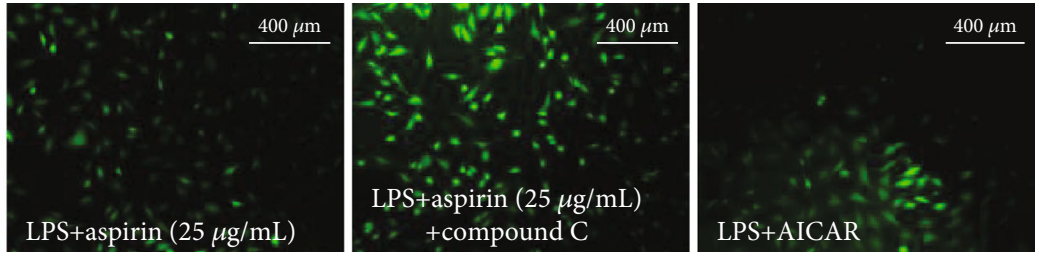

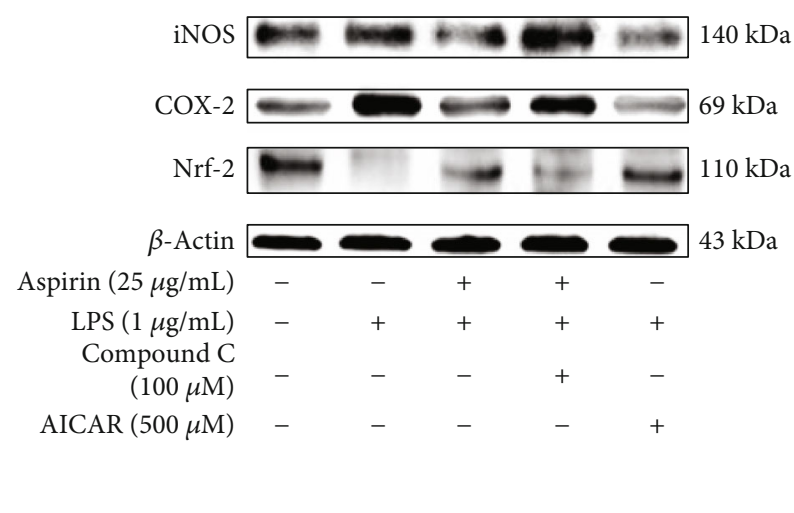

(c)

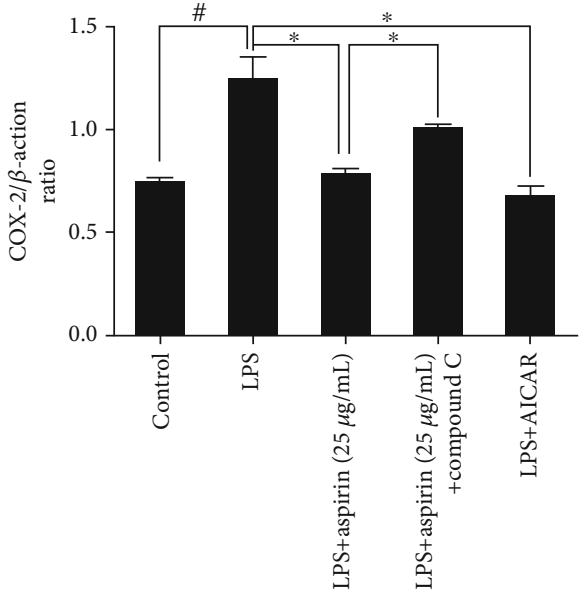

(e)

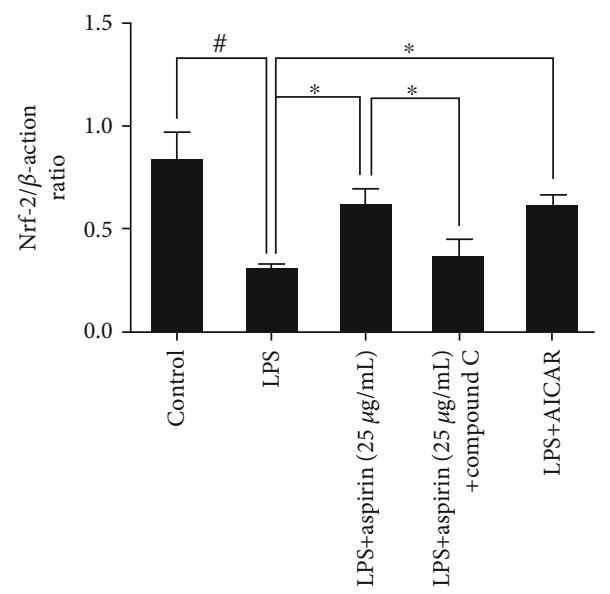

(f)

FIgUre 4: Continued. 


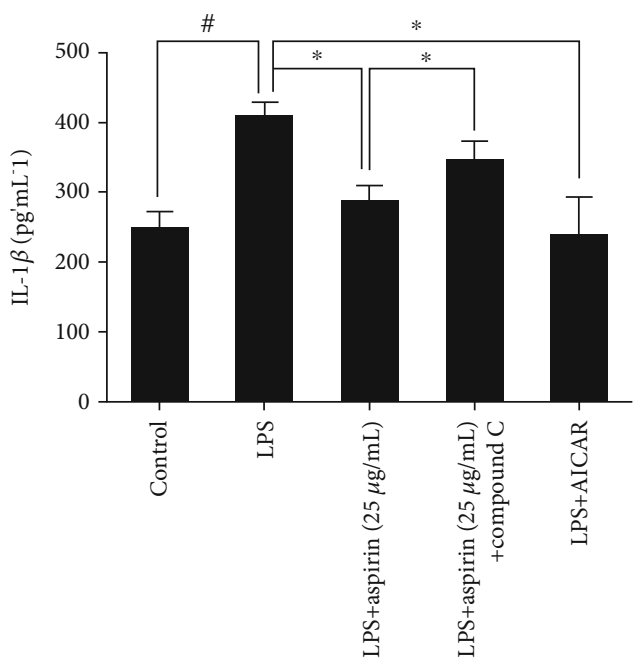

(g)

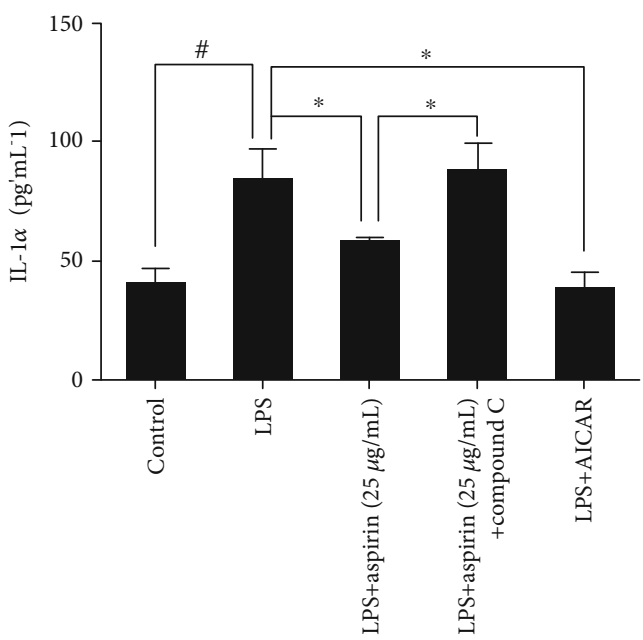

(i)

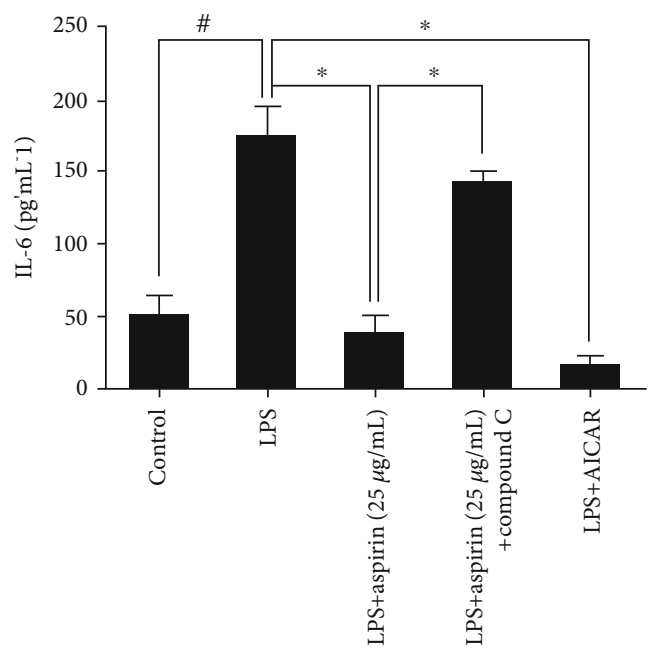

(h)

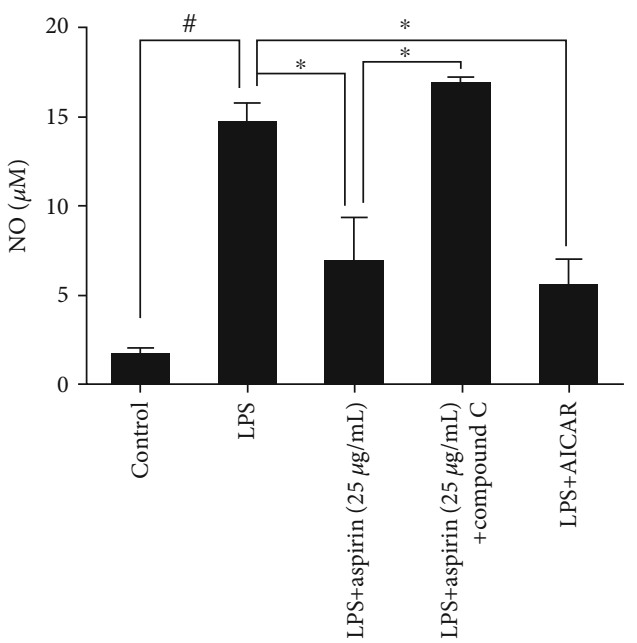

(j)

FIGURE 4: Activation of the APMK signaling pathway is essential to the antioxidative and anti-inflammatory effects of aspirin. NPCs were pretreated with an AMPK inhibitor (compound C, $100 \mu \mathrm{M}$ ) or an AMPK agonist (AICAR, $500 \mu \mathrm{M}$ ) for $24 \mathrm{~h}$; aspirin $(25 \mu \mathrm{g} / \mathrm{mL}$ ) or LPS $(1 \mu \mathrm{g} / \mathrm{mL}$ ) was then added for $24 \mathrm{~h}$. (a) Fluorescence images show ROS levels in different groups. Scale bar, $400 \mu \mathrm{m}$. (b) Average fluorescence intensity of ROS detected by FCM ( ${ }^{\#} P<0.05 v s$. control group; ${ }^{*} P<0.05 v s$. LPS group or aspirin (25 $\left.\mu \mathrm{g} / \mathrm{mL}\right)$ group). (c) Western blotting results for ( $\mathrm{d}-\mathrm{f})$ iNOS, COX-2, and Nrf-2 ( $P<0.05$ vs. control group; ${ }^{*} P<0.05$ vs. LPS group or aspirin $(25 \mu \mathrm{g} / \mathrm{mL})$ group). (g-i) ELISA-detected inflammatory cytokines IL-1 $\beta$, IL-6, and TNF- $\alpha$. (j) NO levels in different groups and ${ }^{*} P$ and ${ }^{*} P<0.05$ by one-way ANOVA and Tukey's HSD test were further analyzed between LPS group $v s$. control group, aspirin $(25 \mu \mathrm{g} / \mathrm{mL}) \mathrm{group} v s$. LPS group, aspirin $(25 \mu \mathrm{g} / \mathrm{mL})+\mathrm{LPS}+$ compound C group $v s$. aspirin $(25 \mu \mathrm{g} / \mathrm{mL})$ group, and LPS+AICAR group vs. LPS group ( $n=3$ independent experiments).

group had many notochordal cells (vacuole-like cells) and NPCs (chondrocyte-like cells). In the IDD group, the demonstrated significantly degenerative changes were observed after puncture. The masses of notochordal cells in the nucleus pulposus were lost, and the nucleus pulposus tissue was subsequently occupied by disorganized hypocellular fibrocartilaginous tissue. Treatment with aspirin, especially high-dose aspirin, significantly inhibited the loss of nucleus pulposus tissue and alleviated the destruction of structures in IVDs compared with the IDD group (Figures $7(\mathrm{~g})$ and $7(\mathrm{~h})$ ).

By IHC staining (Figure 8(a)), we measured the expression of iNOS and COX-2 (Figures $8(\mathrm{~b})$ and $8(\mathrm{c})$ ), which are associated with oxidative stress, and inflammatory cytokines, including IL- $1 \beta$ and TNF- $\alpha$ (Figures $8(\mathrm{~d})$ and $8(\mathrm{e})$ ). All of these were attenuated by aspirin treatment. Notably, iNOS was slightly expressed in the control group but expression levels dramatically increased in the IDD group. Meanwhile, aspirin protected COL2 synthesis and prevented MMP-3 expression compared to the IDD group (Figures $8(\mathrm{f})$ and $8(\mathrm{~g})$ ).

\section{Discussion}

In the present study, we investigated whether aspirin could attenuate degeneration of NPCs due to LPS-induced oxidative stress and inflammation. The results showed that the 


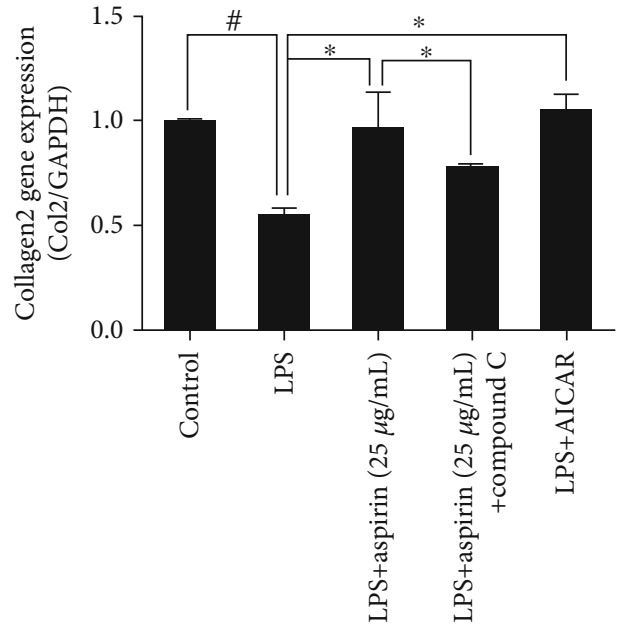

(a)

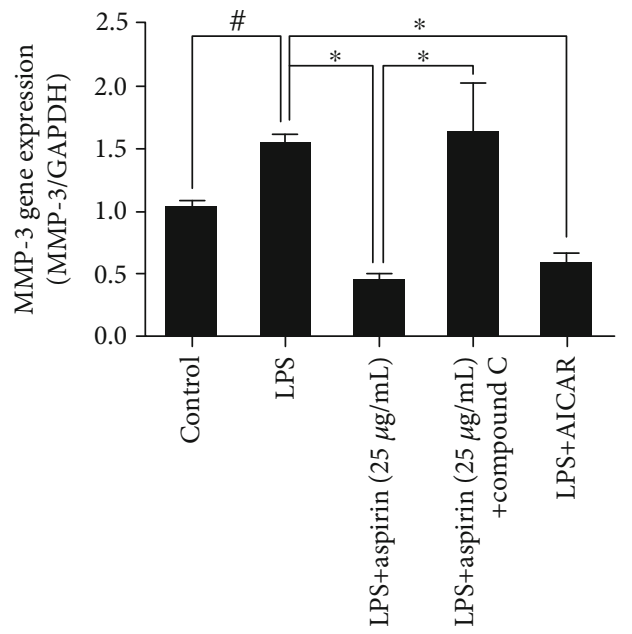

(c)

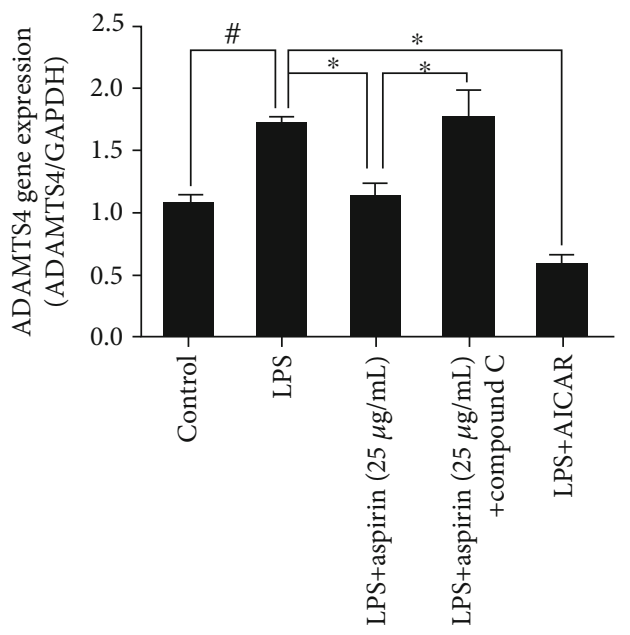

(e)

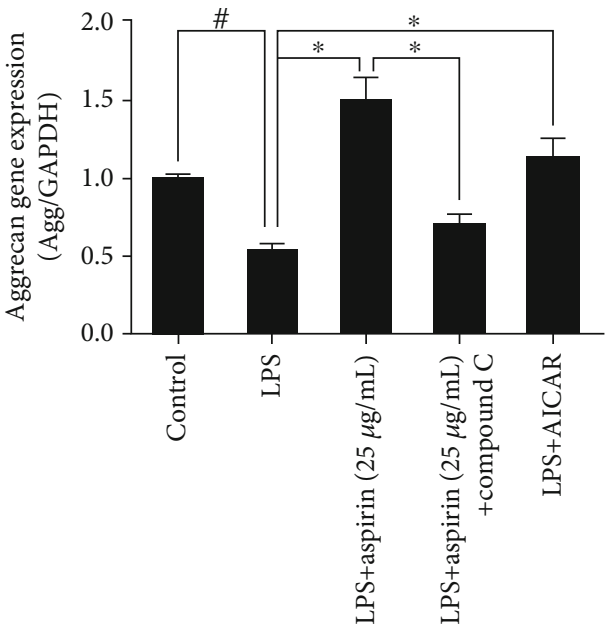

(b)

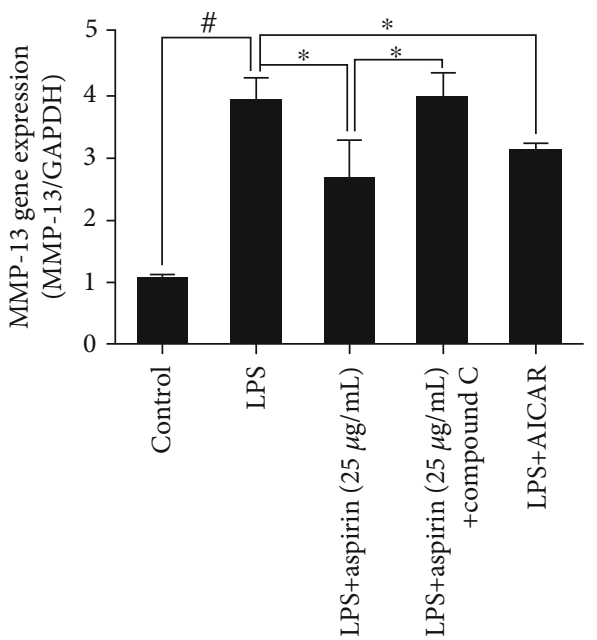

(d)

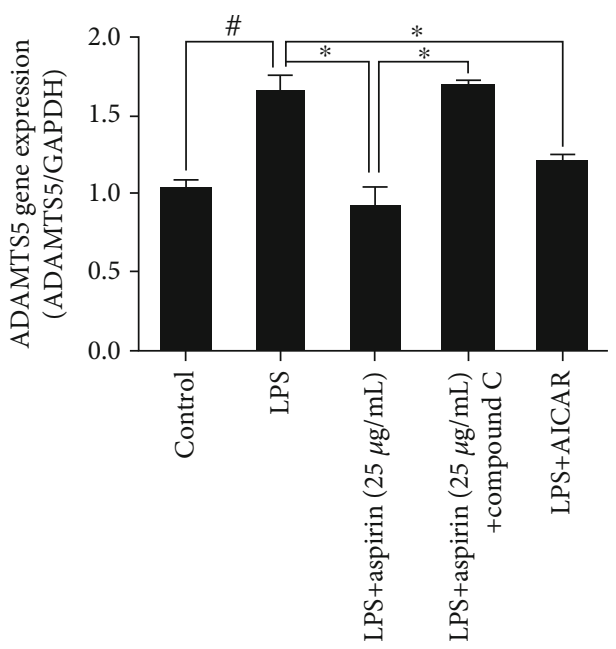

(f)

FIGURE 5: Inhibition of the APMK signaling pathway reverses the protective effects of aspirin on NPC dysfunction. NPCs were pretreated with an AMPK inhibitor (compound C, $100 \mu \mathrm{M}$ ) or an AMPK agonist (AICAR, $500 \mu \mathrm{M})$ for $24 \mathrm{~h}$; aspirin $(25 \mu \mathrm{g} / \mathrm{mL})$ or LPS $(1 \mu \mathrm{g} / \mathrm{mL})$ was then added for $24 \mathrm{~h}$. Subsequently, we obtained RT-PCR results for (a-f) COL2, aggrecan, MMP-3, MMP-13, ADAMTS-4, and ADAMTS-5 (\# $P<0.05$ vs. control group; ${ }^{*} P<0.05 v s$. LPS group or aspirin $(25 \mu \mathrm{g} / \mathrm{mL})$ group). LPS group $v s$. control group, aspirin $(25 \mu \mathrm{g} / \mathrm{mL})$ group $v s$. LPS group, aspirin $(25 \mu \mathrm{g} / \mathrm{mL})+\mathrm{LPS}+$ compound C group $v s$. aspirin $(25 \mu \mathrm{g} / \mathrm{mL})$ group, and LPS + AICAR group $v s$. LPS group $(n=3$ independent experiments). 


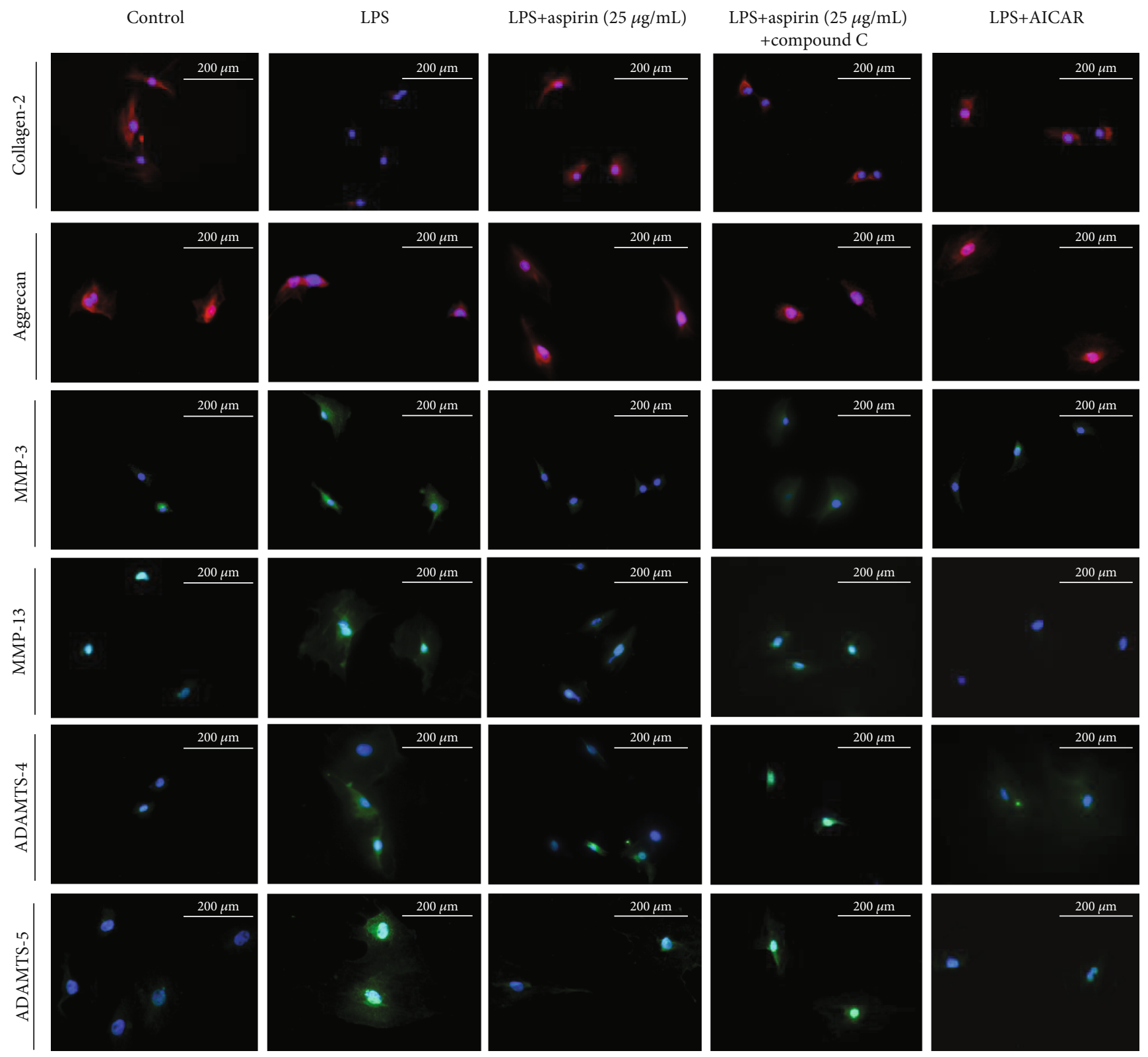

FIGURE 6: ICC staining after application of AMPK pathway agonist and inhibitor. NPCs were pretreated with an AMPK inhibitor (compound C, $100 \mu \mathrm{M})$ or an AMPK agonist (AICAR, $500 \mu \mathrm{M})$ for $24 \mathrm{~h}$; aspirin $(25 \mu \mathrm{g} / \mathrm{mL})$ or LPS $(1 \mu \mathrm{g} / \mathrm{mL})$ was then added for $24 \mathrm{~h}$. Subsequently, we obtained ICC results for (a) representative ICC image with COL2, aggrecan, MMP-3, MMP-13, ADAMTS-4, and ADAMTS-5 staining in NPCs. Scale bar, $200 \mu \mathrm{m}$.

protective mechanism of aspirin involves the activation of the AMPK signaling pathway that attenuates oxidative stress.

Aspirin has been widely used in the clinic as a nonsteroidal anti-inflammatory drug (NSAID) [21]. It has a long history of availability, and its lower cost and increased safety also make it the first-line treatment by numerous physicians. Usually, aspirin is taken to relieve inflammation or mild to moderate pain or fever. It inhibits the aggregation of platelets and reduces the occurrence rate of coronary-artery thrombosis or myocardial infarction [35]. Some epidemiological studies have suggested that long-term use of low-dose aspirin might prevent cancer [36]. Numerous studies have revealed that aspirin may have more potential applications. Piazza et al. found that NSAIDs could induce cell apoptosis and alter the expression of cell cycle regulatory genes [37, 38]. Redlak et al. [39] reported that aspirin could inhibit the activity of protein kinase $\mathrm{C}$, an upstream regulator of activation of reduced $\beta$-nicotinamide adenine dinucleotide $2^{\prime}$-phosphate (NADPH) oxidases. In this study, we found that aspirin could activate AMPK to attenuate oxidative stress and inhibit NPC dysfunction. These findings indicate that aspirin might be considered as a novel option for mitigating IDD.

Oxidative stress is an important factor in the progression of IDD $[12,13]$. Studies have shown that factors, such as 

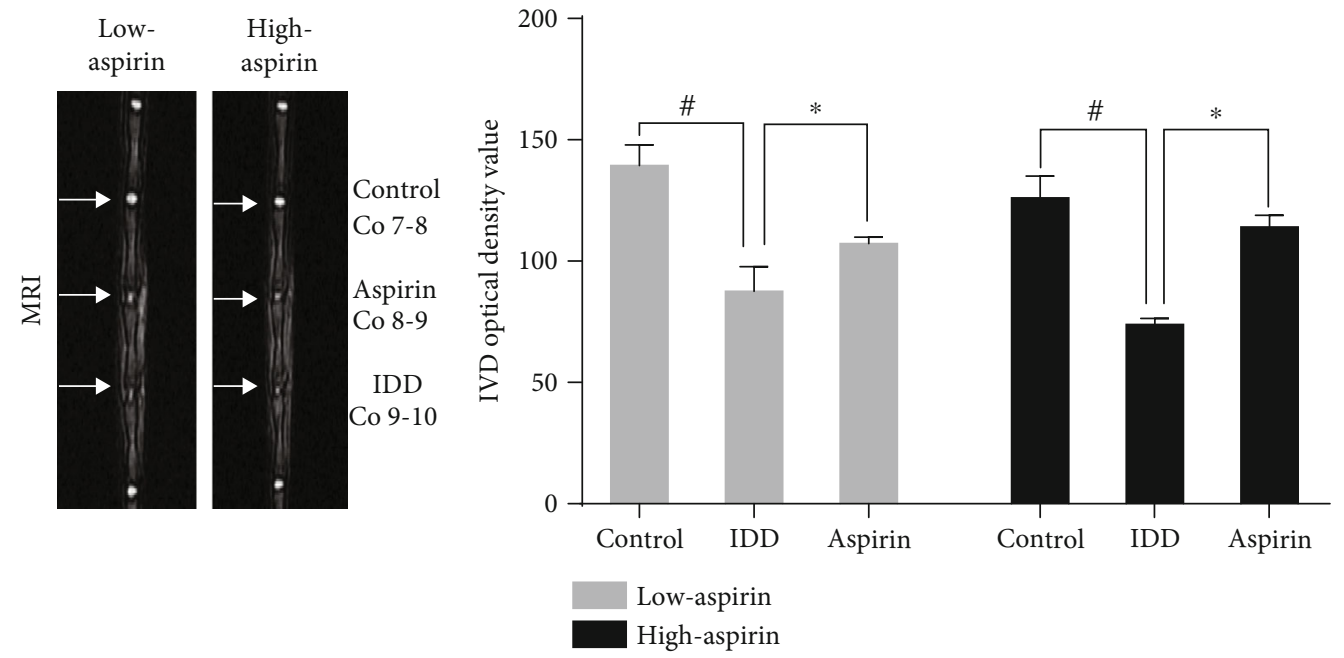

(a)

(b)
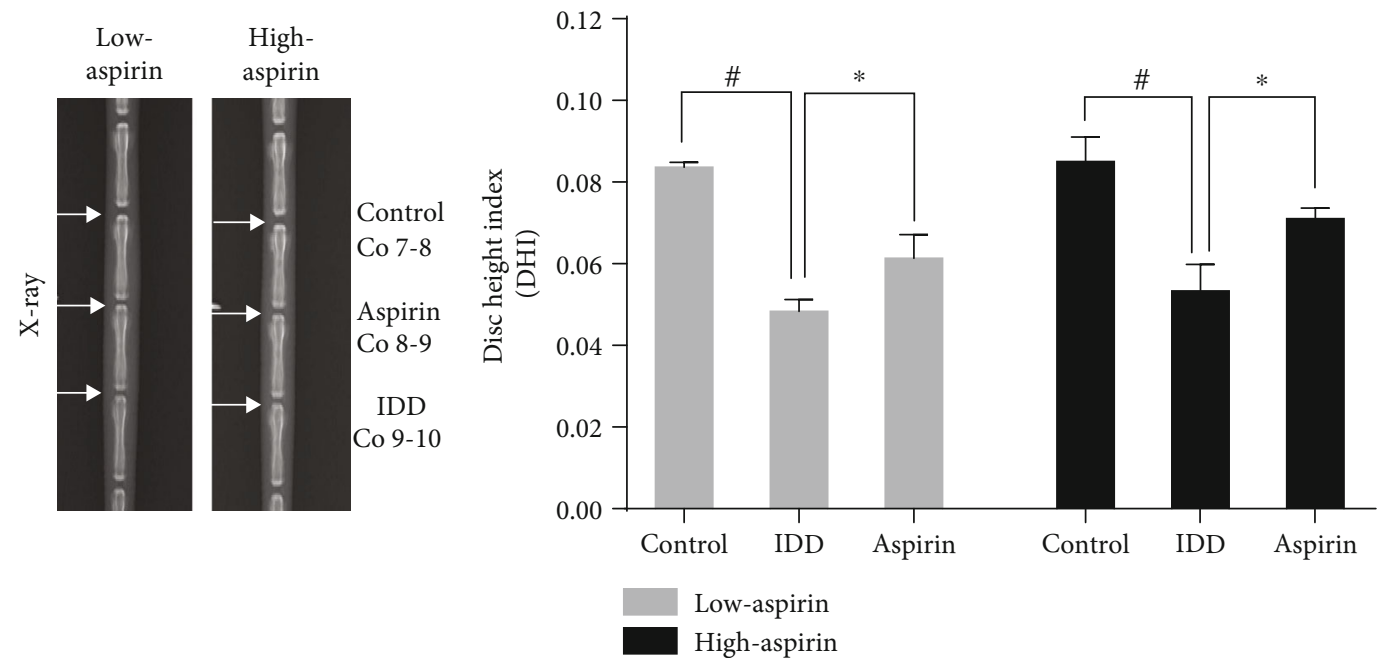

(c)

(d)

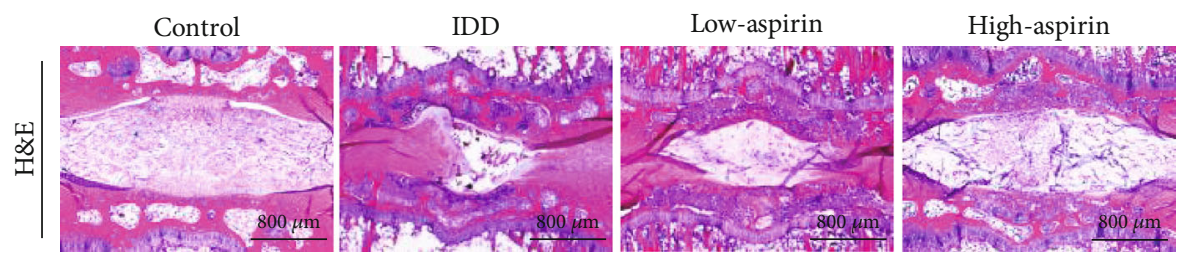

(e)

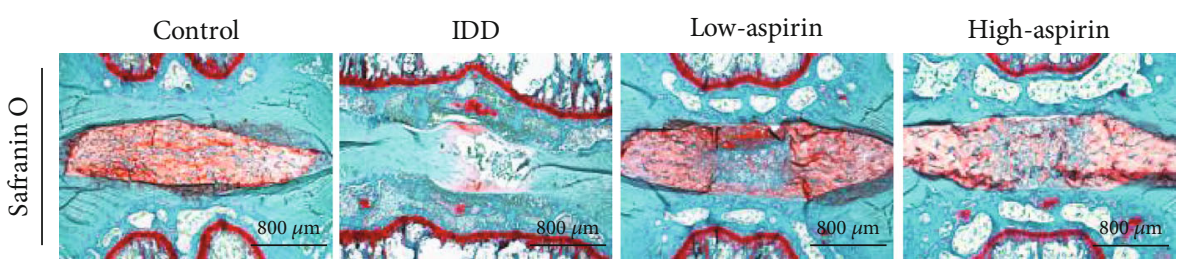

(f)

Figure 7: Continued. 


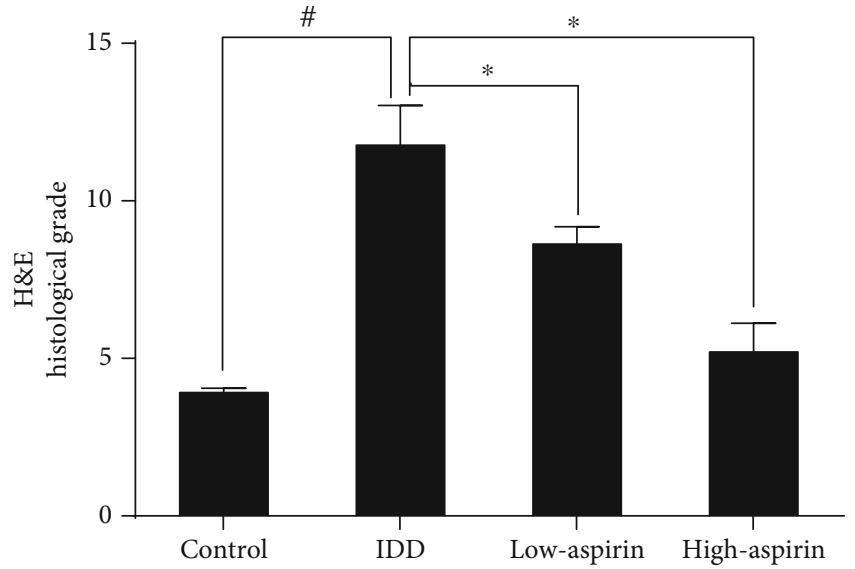

(g)

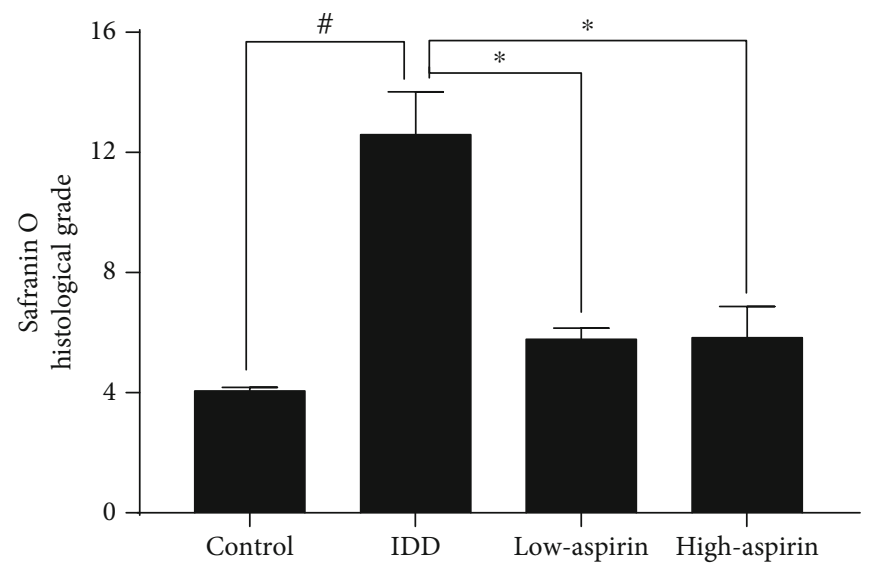

(h)

Figure 7: Aspirin alleviates IDD in vivo. SD rats' coccygeal vertebrae Co7-Co8 discs as the control group and Co8-Co10 were punctured, while those with punctured Co8-Co9 received aspirin (low dose: $n=10$; high dose: $n=10$ ) as aspirin group and punctured Co9-Co10 received PBS as the IDD group. (a) Representative MRI shows IVD signal intensity. (b) Quantitative statistics for IVD optical density (OD) value ( ${ }^{\#} P<0.05$ vs. control group; ${ }^{*} P<0.05$ vs. IDD group). (c) Representative X-ray image. (d) DHI ( $P<0.05$ vs. control group; * $P<0.05$ vs. IDD group). Representative and histological scores of (e) H\&E and (f) Safranin O Fast Green. (g) Quantitative statistics for $\mathrm{H} \& \mathrm{E}$ and (h) Safranin O Fast Green $\left({ }^{\#} P<0.05\right.$ vs. control group; ${ }^{*} P<0.05$ vs. IDD group). Scale bar, $800 \mu \mathrm{m}$. ${ }^{\#} P$ and ${ }^{*} P<0.05$ by oneway ANOVA and Tukey's HSD test were further analyzed between IDD group vs. control group, low-aspirin group vs. IDD group, highaspirin group $v s$. IDD group ( $n=10$ independent experiments).

aging or cellular senescence, malnutrition, and some diseases, such as type II diabetes, could induce oxidative stress in IVDs [40-42]. Notably, NPCs are in hypoxic environments and gain energy mainly through glycolysis [16]. These produce ROS via oxidative metabolism, but overproduction of ROS directly induces damage to NPCs and perturbs the homeostasis of the disc matrix. For example, ROS may reduce synthesis of proteoglycans and increase MMP expression [43]. Moreover, many proinflammatory cytokines can significantly stimulate production of ROS, especially in degenerated or aged IVDs [16]. In addition, NO is an important inflammatory modulator in osteoarthritic cartilage. Earlier studies have shown that reducing NO production by inhibiting iNOS can significantly reduce production of important catabolic factors, such as metalloproteinases and IL- $1 \beta$ [44]. In our study, aspirin significantly decreased iNOS and NO, which indicates that it attenuates oxidative stress in IDD.

AMPK is a serine/threonine protein kinase that is uniquely expressed in mammalian cells. It participates in many biological functions, such as regulating energy balance and cell metabolism [45]. The AMPK signaling pathway has been extensively studied in metabolic diseases, such as diabetes. Some researchers have found that AMPK mediates energy metabolism at the intracellular level and can suppress various pathological oxidative stressors or inflammatory responses [46]. Our results showed that aspirin could activate the AMPK signaling pathway in NPCs. Recently, Hawley et al. [47] found that salicylate inhibits dephosphorylation of the activating phosphorylation site threonine-172 and activates the AMPK signaling pathway, which agrees with our results. In addition, we found that preinhibition of the
AMPK pathway reversed the protective effects of aspirin on inflammation, oxidation, and thereby NPC dysfunction, which further demonstrates that aspirin attenuates IVD by activating the AMPK signaling pathway. To our knowledge, this is the first study that has shown that aspirin can attenuate oxidative stress in NPCs through this pathway. Hawley et al. [47] also found that salicylate activates AMPK and bound to the same site at which a synthetic activator (A-769662) induces allosteric activation of AMPK. We have shown that the activity of the AMPK signaling pathway leads to a significant decrease in the downstream production of oxidative stressors, including ROS and iNOS. Meanwhile, through the inhibition of the AMPK signaling pathway, compound C promotes oxidative stress [48] and increases ROS expression [49].

We observed an interesting and notable phenomenon in our current study: increased iNOS led to an increase in downstream output of NO, and activating AMPK attenuated the expression of iNOS. This finding is the same as those of Tsoyi et al. [50] and Chen et al. [51] in NPCs but is in contrast with the results of Lee et al. [52], who found that AMPK promotes the expression of iNOS and production of $\mathrm{NO}$ in kidney proximal tubular epithelial cells. Their results seemed to show that iNOS is a positive factor that protects cells from an oxidative stress environment. However, iNOS inhibition did not slow the progression of osteoarthritis in human patients. In the current study, we observed that the application of a high dose of aspirin significantly attenuated the production of iNOS, NO, and ROS and subsequently suppressed NPC dysfunction in vitro and reverses the IDD process in vivo. We hypothesize that the pathogenic mechanisms of 

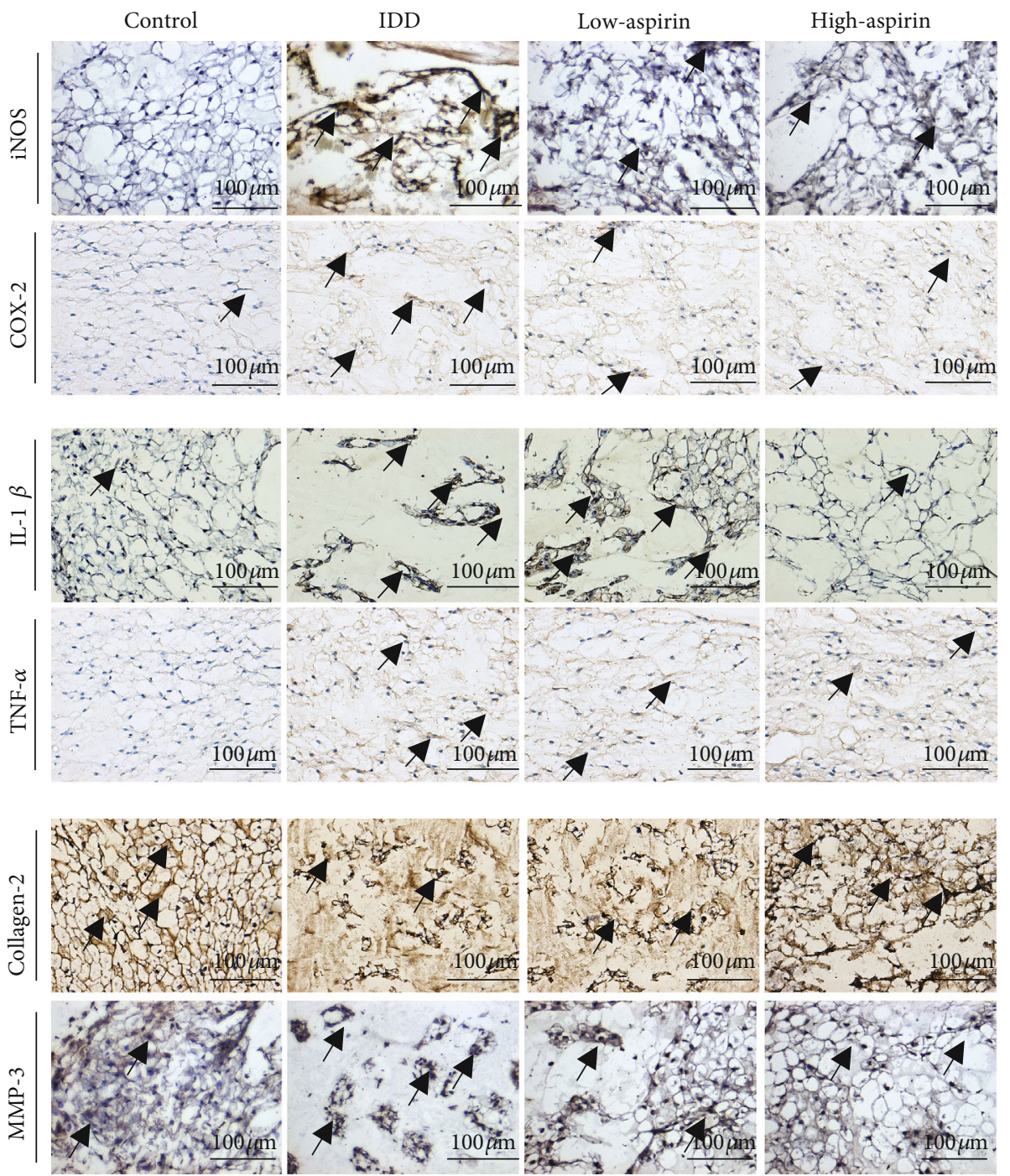

(a)

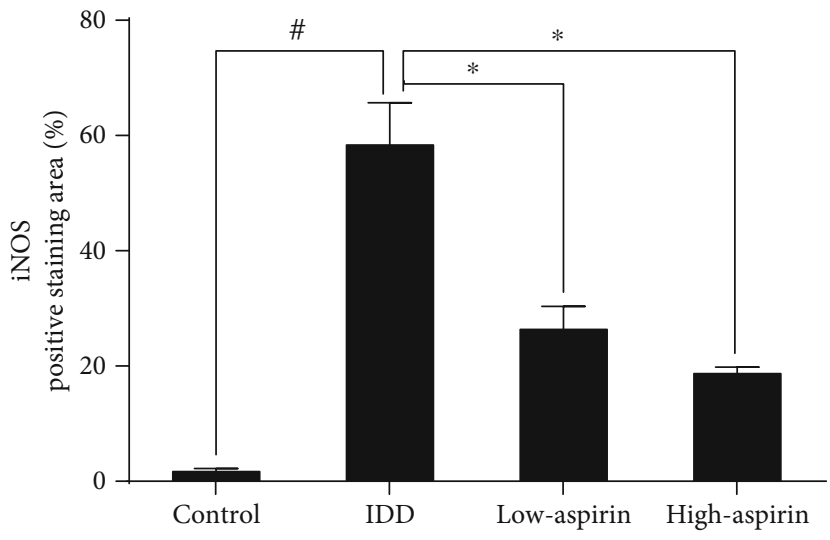

(b)

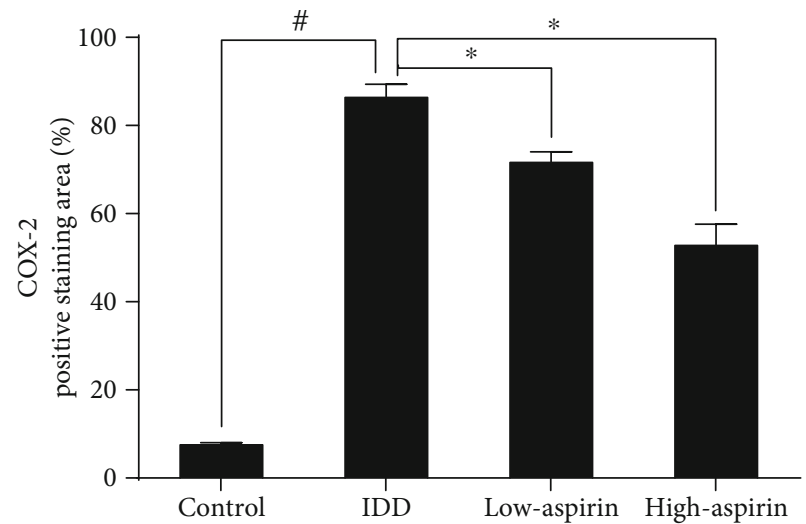

(c)

Figure 8: Continued. 


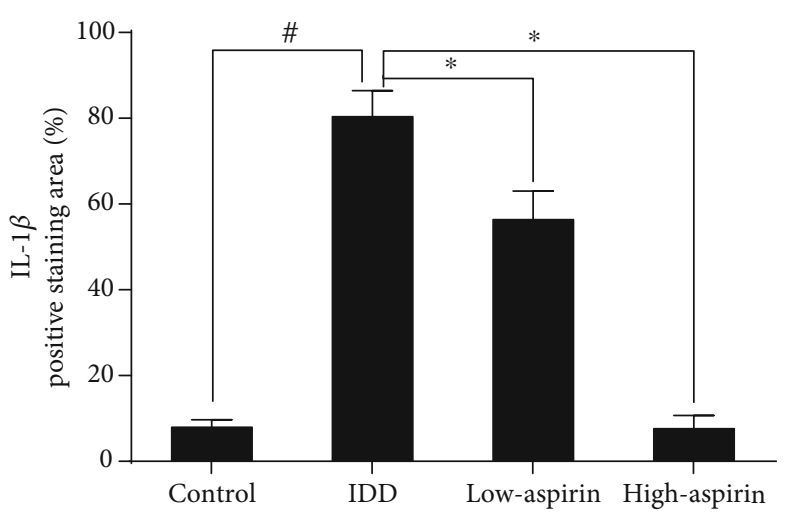

(d)

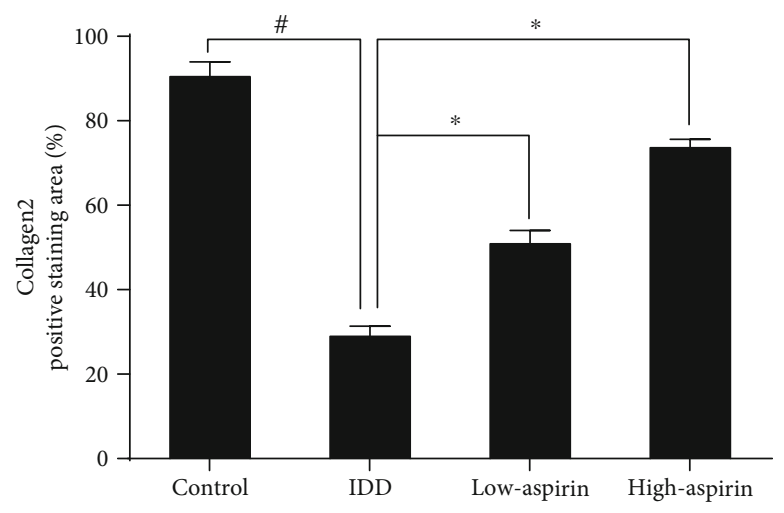

(f)

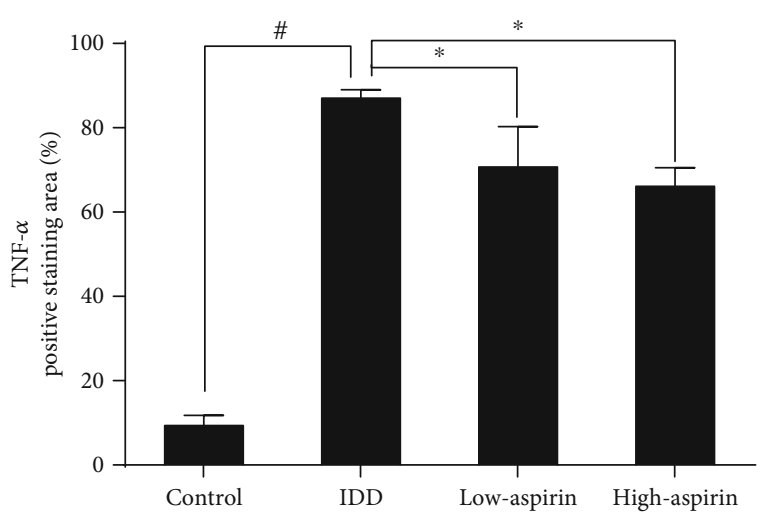

(e)

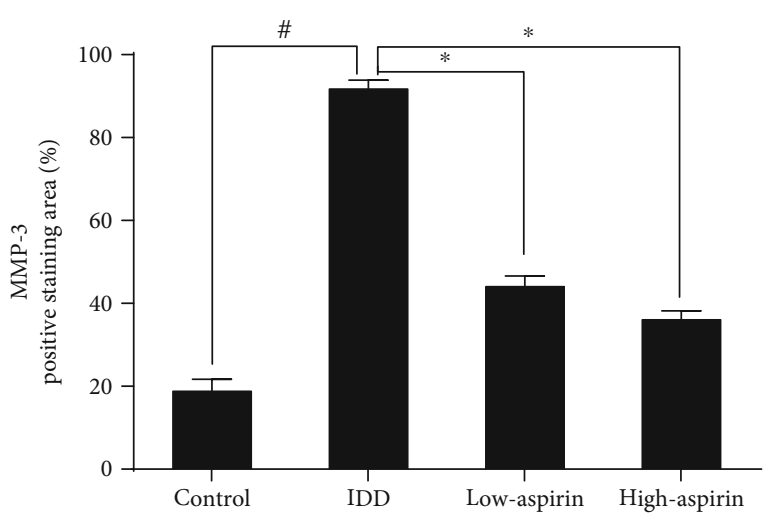

(g)

FIGURE 8: IHC staining in vivo. (a) Representative specimens of paraffin section with IHC staining of iNOS, COX-2, IL- $1 \beta$, TNF- $\alpha$, COL2, and MMP-3 in nucleus pulposus tissue; scale bar, $100 \mu \mathrm{m}$. (b-g) The quantitative statistics for iNOS, COX-2, IL- $1 \beta$, TNF- $\alpha$, COL2, and MMP-3 staining ( $P<0.05$ vs. control group; ${ }^{*} P<0.05$ vs. IDD group). ${ }^{\#} P$ and ${ }^{*} P<0.05$ by one-way ANOVA and Tukey's HSD test were further analyzed between IDD group $v s$. control group, low-aspirin group $v s$. IDD group, and high-aspirin group $v s$. IDD group $(n=10$ independent experiments).

osteoarthritis might be different from those of IDD and iNOS might play different physiological roles in various cell lines.

\section{Conclusions}

Here, we demonstrated that aspirin activates the AMPK signaling pathway to ameliorate the process of IVD deterioration. Oxidative stress is an important pathological change in IDD, and effectively relieving it could potentially be a novel target for the treatment of IDD.

\section{Data Availability}

The data used to support the findings of this study are available from the corresponding authors upon request.

\section{Conflicts of Interest}

The authors declare no conflict of interest.

\section{Authors' Contributions}

Yu Liu, Jiayi Lin, and Xiexing Wu contributed equally to this work.

\section{Acknowledgments}

This research was supported by the National Nature Science Foundation of China (81873990, 81873991, 81472077, 81672238,81372018 , and 81972104), the Jiangsu Provincial Medical Youth Talent (QNRC2016751), the Natural Science Foundation of Jiangsu Province (BK20180001), the application of key technology research program of Suzhou City (SS201858), and the Project Funded by the Priority Academic Program Development of Jiangsu Higher Education Institutions (PAPD).

\section{Supplementary Materials}

RT-PCR detection of the applied primer sequences. Primer sequence design by querying the NCBI database. Supplementary Figure 1: LPS induced oxidative stress and degeneration in NPCs. (A) We cultured NPCs in different concentrations of LPS $(0,0.01,0.1,1$, and $10 \mu \mathrm{g} / \mathrm{mL})$ for 1,3 , and 5 days and then detected cell viability by CCK- $8\left({ }^{*} P<0.05\right.$ vs. control group). (B) Fluorescence images showed ROS levels in different groups. Scale bar, $400 \mu \mathrm{m}$. (C, D) Average fluorescence intensity of ROS was detected by FCM $\left({ }^{*} P<0.05\right.$ vs. control group). (C) The NPCs were cultured in different 
concentrations of LPS $(0,0.01,0.1$, and $1 \mu \mathrm{g} / \mathrm{mL})$ for 24 hours. (D) The NPCs were cultured with LPS $(1 \mu \mathrm{g} / \mathrm{mL})$ in different hours $(0,0.25,0.5,1,3,6,12$, and 24 hours $)$. NPCs were pretreated with LPS $(1 \mu \mathrm{g} / \mathrm{mL})$ for $12 \mathrm{~h}$. Subsequently, we observed ICC staining of COL2 and MMP-3 in NPCs. (E) Representative image of ICC staining of COL2 and MMP-3 in NPCs. Scale bar, $200 \mu \mathrm{m}$. (F) Western blot results of iNOS, COX-2, and Nrf-2 and (G-I) the quantitative statistic, respectively ( ${ }^{*} \mathrm{P}<0.05$ vs. control group). (J-M) RT-PCR results of COL2, aggrecan, iNOS, and COX-2 ( ${ }^{*} P<0.05$ vs. control group; N.S. $=$ no statistical significance). ${ }^{*} P<0.05$ by one-way ANOVA and Tukey's HSD tests was further analyzed between each group vs. control $(n=3$ independent experiments). Supplementary Figure 2: the effect of compound $\mathrm{C}$ or AICAR on the AMPK signaling pathway. NPCs were treated solely with an AMPK inhibitor (compound C, $100 \mu \mathrm{M}$ ) or an AMPK agonist (AICAR, $500 \mu \mathrm{M}$ ) for $24 \mathrm{~h}$. (A, B) Western blot results of p-AMPK, AMPK, p-ACC, and ACC. (C, D) Densitometric analysis of p-AMPK/AMPK and $\mathrm{p}$-ACC/ACC levels, respectively $\left({ }^{*} P<0.05\right.$ vs. control group). ${ }^{*} P<0.05$ by one-way ANOVA and Tukey's HSD tests was further analyzed between AICAR group and compound $\mathrm{C}$ group vs. control group ( $n=3$ independent experiments). (Supplementary Materials)

\section{References}

[1] B. F. Walker, "The prevalence of low back pain: a systematic review of the literature from 1966 to 1998," Journal of Spinal Disorders, vol. 13, no. 3, pp. 205-217, 2000.

[2] T. Driscoll, G. Jacklyn, J. Orchard et al., "The global burden of occupationally related low back pain: estimates from the Global Burden of Disease 2010 study," Annals of the Rheumatic Diseases, vol. 73, no. 6, pp. 975-981, 2014.

[3] W. T. Crow and D. R. Willis, "Estimating cost of care for patients with acute low back pain: a retrospective review of patient records," The Journal of the American Osteopathic Association, vol. 109, no. 4, pp. 229-233, 2009.

[4] S. K. Mirza and R. A. Deyo, "Systematic review of randomized trials comparing lumbar fusion surgery to nonoperative care for treatment of chronic back pain," Spine, vol. 32, no. 7, pp. 816-823, 2007.

[5] R. H. Pearce, B. J. Grimmer, and M. E. Adams, "Degeneration and the chemical composition of the human lumbar intervertebral disc," Journal of Orthopaedic Research, vol. 5, no. 2, pp. 198-205, 1987.

[6] K. Luoma, H. Riihimäki, R. Luukkonen, R. Raininko, E. Viikari-Juntura, and A. Lamminen, "Low back pain in relation to lumbar disc degeneration," Spine, vol. 25, no. 4, pp. 487-492, 2000.

[7] J. A. Buckwalter, "Aging and degeneration of the human intervertebral disc," Spine, vol. 20, no. 11, pp. 1307-1314, 1995.

[8] J. Wang, D. Markova, D. G. Anderson, Z. Zheng, I. M. Shapiro, and M. V. Risbud, "TNF- $\alpha$ and IL- $1 \beta$ promote a disintegrinlike and metalloprotease with thrombospondin type I motif5 -mediated aggrecan degradation through syndecan-4 in intervertebral disc," Journal of Biological Chemistry, vol. 286, no. 46, pp. 39738-39749, 2011.

[9] Z. I. Johnson, Z. R. Schoepflin, H. Choi, I. M. Shapiro, and M. V. Risbud, "Disc in flames: roles of TNF- $\alpha$ and IL- $1 \beta$ in intervertebral disc degeneration," European Cells \& Materials, vol. 30, pp. 104-116, 2015.

[10] M. Grönblad, J. Virri, J. Tolonen et al., “A controlled immunohistochemical study of inflammatory cells in disc herniation tissue," Spine, vol. 19, no. 24, pp. 2744-2751, 1994.

[11] B. E. Bachmeier, A. G. Nerlich, C. Weiler, G. Paesold, M. Jochum, and N. Boos, "Analysis of tissue distribution of TNF- $\alpha$, TNF- $\alpha$-receptors, and the activating TNF- $\alpha$-converting enzyme suggests activation of the TNF- $\alpha$ system in the aging intervertebral disc," Annals of the New York Academy of Sciences, vol. 1096, no. 1, pp. 44-54, 2010.

[12] J. W. Chen, B. B. Ni, B. Li, Y. H. Yang, S. D. Jiang, and L. S. Jiang, "The responses of autophagy and apoptosis to oxidative stress in nucleus pulposus cells: implications for disc degeneration," Cellular Physiology and Biochemistry, vol. 34, no. 4, pp. 1175-1189, 2014.

[13] J. Chen, J. Xuan, Y. T. Gu et al., "Celastrol reduces IL-1 $\beta$ induced matrix catabolism, oxidative stress and inflammation in human nucleus pulposus cells and attenuates rat intervertebral disc degeneration in vivo," Biomedicine \& Pharmacotherapy, vol. 91, pp. 208-219, 2017.

[14] A. Dimozi, E. Mavrogonatou, A. Sklirou, and D. Kletsas, "Oxidative stress inhibits the proliferation, induces premature senescence and promotes a catabolic phenotype in human nucleus pulposus intervertebral disc cells," European Cells \& Materials, vol. 30, pp. 89-103, 2015.

[15] H. Zhang, X. Kong, J. Kang et al., "Oxidative stress induces parallel autophagy and mitochondria dysfunction in human glioma U251 cells," Toxicological Sciences, vol. 110, no. 2, pp. 376-388, 2009.

[16] L. A. Nasto, A. R. Robinson, K. Ngo et al., "Mitochondrialderived reactive oxygen species (ROS) play a causal role in aging-related intervertebral disc degeneration," Journal of Orthopaedic Research, vol. 31, no. 7, pp. 1150-1157, 2013.

[17] Y. H. Cheng, S. H. Yang, and F. H. Lin, “Thermosensitive chitosan-gelatin-glycerol phosphate hydrogel as a controlled release system of ferulic acid for nucleus pulposus regeneration," Biomaterials, vol. 32, no. 29, pp. 6953-6961, 2011.

[18] J. Egea, I. Fabregat, Y. M. Frapart et al., "European contribution to the study of ROS: a summary of the findings and prospects for the future from the COST action BM1203 (EUROS)," Redox Biology, vol. 13, pp. 94-162, 2017.

[19] M. Manish, M. R. Siddiqui, K. Tran, S. P. Reddy, and A. B. Malik, "Reactive oxygen species in inflammation and tissue injury," Antioxidants \& Redox Signaling, vol. 20, no. 7, pp. 1126-1167, 2014.

[20] M. H. Dreser, "Pharmakologisches über aspirin (Acetylsalicylsäure)," Archiv Für Die Gesamte Physiologie Des Menschen Und Der Tiere, vol. 76, no. 5-6, pp. 306-318, 1970.

[21] E. V. P. Espinosa, J. P. Murad, and F. T. Khasawneh, “Aspirin: pharmacology and clinical applications," Thrombosis, vol. 2012, no. 5, Article ID 626289, 1 pages, 2012.

[22] N. O'Gorman, D. Wright, D. L. Rolnik, K. H. Nicolaides, and L. C. Poon, "Study protocol for the randomised controlled trial: combined multimarker screening and randomised patient treatment with ASpirin for evidence-based PREeclampsia prevention (ASPRE)," BMJ Open, vol. 6, no. 6, article e011801, 2016.

[23] S. Rachidi, A. Metelli, B. Riesenberg et al., "Platelets subvert T cell immunity against cancer via GARP-TGF $\beta$ axis," Science Immunology, vol. 2, no. 11, article eaai7911, 2017. 
[24] Y. P. Wang, Y. Wu, L. Y. Li et al., “Aspirin-triggered lipoxin A4 attenuates LPS-induced pro-inflammatory responses by inhibiting activation of NF- $\kappa \mathrm{B}$ and MAPKs in BV-2 microglial cells," Journal of Neuroinflammation, vol. 8, no. 1, p. 95, 2011.

[25] Z. Wang, W. Huang, and Z. Zuo, "Perioperative aspirin improves neurological outcome after focal brain ischemia possibly via inhibition of notch 1 in rat," Journal of Neuroinflammation, vol. 11, no. 1, p. 56, 2014.

[26] R. He, M. Cui, H. Lin et al., "Melatonin resists oxidative stressinduced apoptosis in nucleus pulposus cells," Life Sciences, vol. 199, pp. 122-130, 2018.

[27] G. Gong, T. Dang, Y. Deng et al., "Physicochemical properties and biological activities of polysaccharides from Lycium barbarum prepared by fractional precipitation," International Journal of Biological Macromolecules, vol. 109, pp. 611-618, 2017.

[28] P. D. Siebert, "Rt-PCR methods and applications," Methods in Molecular Medicine, vol. 13, no. 4, pp. 29-53, 1998.

[29] B. Han, K. Zhu, F. C. Li et al., "A simple disc degeneration model induced by percutaneous needle puncture in the rat tail," Spine, vol. 33, no. 18, pp. 1925-1934, 2008.

[30] H. J. Mao, Q. X. Chen, B. Han et al., "The effect of injection volume on disc degeneration in a rat tail model," Spine, vol. 36, no. 16, pp. E1062-E1069, 2011.

[31] X. Wu, Y. Liu, X. Guo et al., "Prolactin inhibits the progression of intervertebral disc degeneration through inactivation of the NF- $\kappa$ B pathway in rats," Cell Death \& Disease, vol. 9, no. 2, p. 98, 2018.

[32] H. S. An, "A novel rabbit model of mild, reproducible disc degeneration by an anulus needle puncture: correlation between the degree of disc injury and radiological and histological appearances of disc degeneration," Spine, vol. 30, no. 1, pp. 5-14, 2005.

[33] A. Goraca, A. Piechota, and H. Hukkolega, "Effect of alphalipoic acid on LPS-induced oxidative stress in the heart," Journal of Physiology \& Pharmacology, vol. 60, no. 1, pp. 61-68, 2009.

[34] S. Nakagawa, Y. Arai, O. Mazda et al., "N-acetylcysteine prevents nitric oxide-induced chondrocyte apoptosis and cartilage degeneration in an experimental model of osteoarthritis," Journal of Orthopaedic Research, vol. 28, no. 2, pp. 156-163, 2010.

[35] US Preventive Services Task Force, "Aspirin for the prevention of cardiovascular disease: U.S. Preventive Services Task Force recommendation statement," Annals of Internal Medicine, vol. 150, no. 6, pp. 396-404, 2009.

[36] M. S. Reimers, E. Bastiaannet, R. E. Langley et al., "Expression of HLA class I antigen, aspirin use, and survival after a diagnosis of colon cancer," JAMA Internal Medicine, vol. 174, no. 5, p. 732, 2014.

[37] G. A. Piazza, A. L. Rahm, M. Krutzsch et al., "Antineoplastic drugs sulindac sulfide and sulfone inhibit cell growth by inducing apoptosis," Cancer Research, vol. 55, no. 14, pp. 31103116, 1995.

[38] G. A. Piazza, A. K. Rahm, T. S. Finn et al., "Apoptosis primarily accounts for the growth-inhibitory properties of sulindac metabolites and involves a mechanism that is independent of cyclooxygenase inhibition, cell cycle arrest, and p53 induction," Cancer Research, vol. 57, no. 12, pp. 2452-2459, 1997.

[39] M. J. Redlak, J. J. Power, and T. A. Miller, "Prevention of deoxycholate-induced gastric apoptosis by aspirin: roles of
NF- $\kappa \mathrm{B}$ and PKC signaling," Journal of Surgical Research, vol. 145, no. 1, pp. 66-73, 2008.

[40] J. C. Lotz and J. R. Chin, "Intervertebral disc cell death is dependent on the magnitude and duration of spinal loading," Spine, vol. 25, no. 12, pp. 1477-1483, 2000.

[41] G. D. V. Lizette, "Oxidative stress in the aging process: fundamental aspects and new insights," ACS Symposium Series, vol. 2, pp. 177-219, 2015.

[42] R. A. Deyo and J. E. Bass, "Lifestyle and low-back pain. The influence of smoking and obesity," Spine, vol. 14, no. 5, pp. 501-506, 1989.

[43] O. Krupkova, J. Handa, M. Hlavna et al., "The natural polyphenol epigallocatechin gallate protects intervertebral disc cells from oxidative stress," Oxidative Medicine and Cellular Longevity, vol. 2016, Article ID 7031397, 17 pages, 2016.

[44] J. P. Pelletier, D. V. Jovanovic, V. Lascau-Coman et al., "Selective inhibition of inducible nitric oxide synthase reduces progression of experimental osteoarthritis in vivo: possible link with the reduction in chondrocyte apoptosis and caspase 3 level," Arthritis \& Rheumatism, vol. 43, no. 6, pp. 1290-1299, 2010.

[45] D. G. Hardie, J. W. Scott, D. A. Pan, and E. R. Hudson, "Management of cellular energy by the AMP-activated protein kinase system," FEBS Letters, vol. 546, no. 1, pp. 113-120, 2003.

[46] Z. Yang, B. B. Kahn, H. Shi, and B. Xue, "Macrophage $\alpha 1$ AMP-activated protein kinase ( $\alpha 1 \mathrm{AMPK})$ antagonizes fatty acid-induced inflammation through SIRT1," Journal of Biological Chemistry, vol. 285, no. 25, pp. 19051-19059, 2010.

[47] S. A. Hawley, M. D. Fullerton, F. A. Ross et al., "The ancient drug salicylate directly activates AMP-activated protein kinase," Science, vol. 336, no. 6083, pp. 918-922, 2012.

[48] G. Barreto-Torres, J. S. Hernandez, S. Jang et al., "The beneficial effects of AMP kinase activation against oxidative stress are associated with prevention of PPAR $\alpha$-cyclophilin D interaction in cardiomyocytes," American Journal of PhysiologyHeart and Circulatory Physiology, vol. 308, no. 7, pp. H749H758, 2015.

[49] K. Seo, S. Seo, S. H. Ki, and S. M. Shin, "Compound C increases Sestrin2 expression via mitochondria-dependent ROS production," Biological \& Pharmaceutical Bulletin, vol. 39, no. 5, pp. 799-806, 2016.

[50] K. Tsoyi, H. J. Jang, I. T. Nizamutdinova et al., "Metformin inhibits HMGB1 release in LPS-treated RAW 264.7 cells and increases survival rate of endotoxaemic mice," British Journal of Pharmacology, vol. 162, no. 7, pp. 1498-1508, 2011.

[51] D. Chen, D. Xia, Z. Pan et al., "Metformin protects against apoptosis and senescence in nucleus pulposus cells and ameliorates disc degeneration in vivo," Cell Death \& Disease, vol. 7, no. 10, article e2441, 2016.

[52] H. J. Lee, D. Y. Lee, M. M. Mariappan et al., "Hydrogen sulfide inhibits high glucose-induced NADPH oxidase 4 expression and matrix increase by recruiting inducible nitric oxide synthase in kidney proximal tubular epithelial cells," Journal of Biological Chemistry, vol. 292, no. 14, pp. 5665-5675, 2017. 


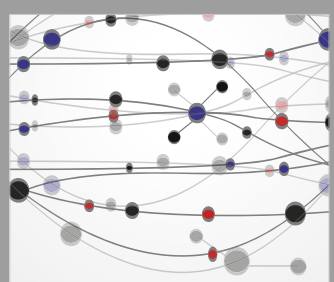

The Scientific World Journal
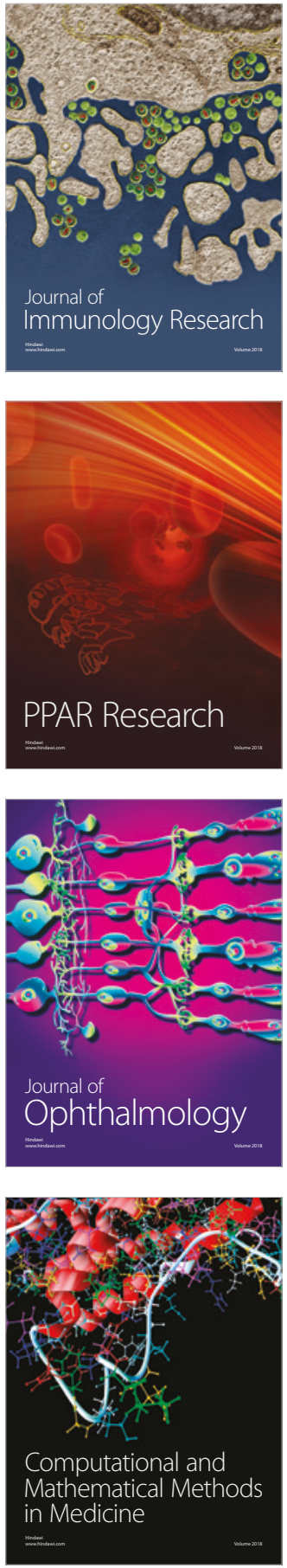

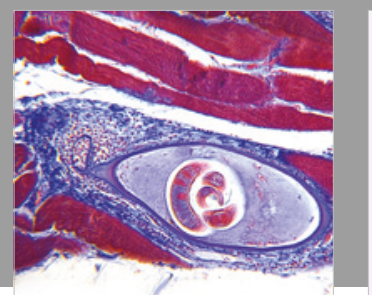

Gastroenterology Research and Practice

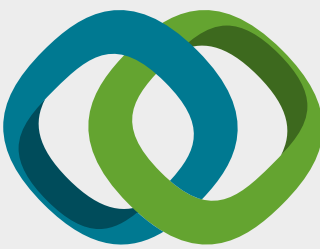

\section{Hindawi}

Submit your manuscripts at

www.hindawi.com
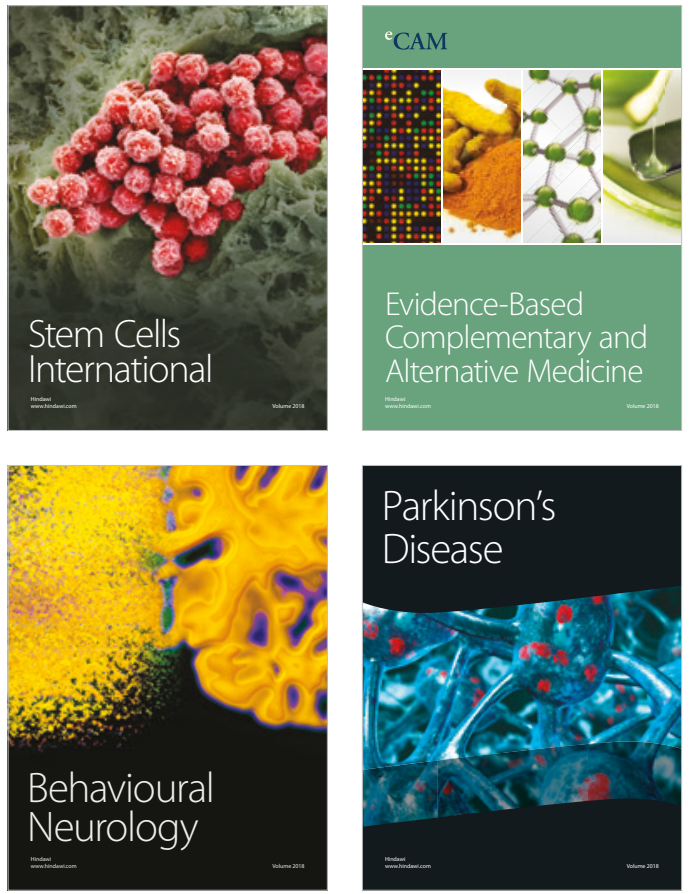

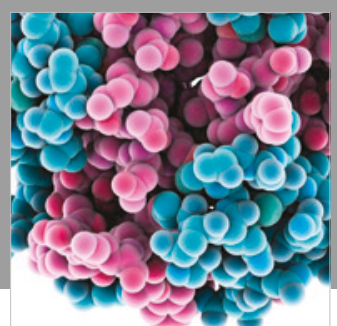

ournal of

Diabetes Research

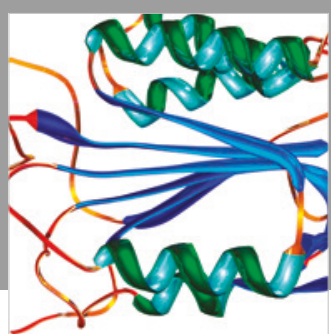

Disease Markers
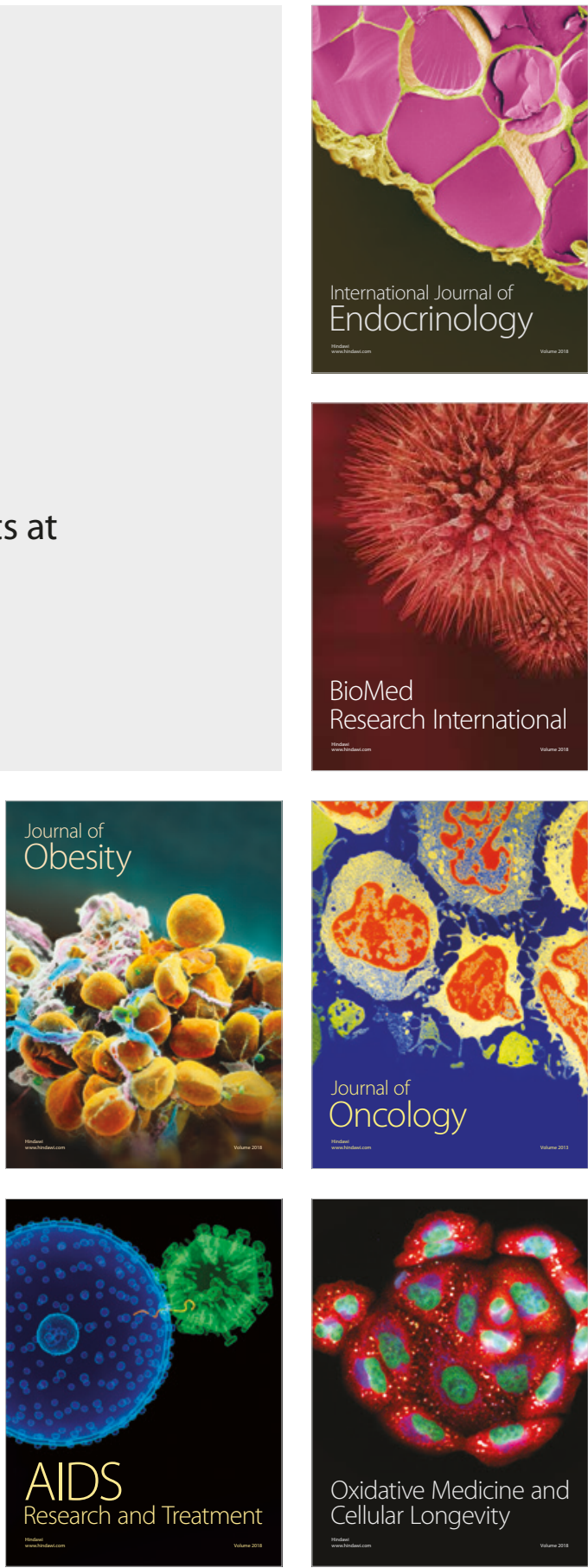